\title{
El sistema de concordancia del participante en las lenguas Pano y sus implicancias para el conocimiento de la Proto-Lengua ${ }^{1}$
}

Pilar M. Valenzuela ${ }^{2}$

\begin{abstract}
Resumen
En las lenguas de la familia Pano una serie de construcciones adjuntas (que expresan lugar, etapa de vida, cuantificación distributiva, manera, entre otros) están semánticamente orientadas hacia uno de los participantes obligatorios de la cláusula. Dicha relación es marcada explícitamente mediante una morfología flexiva al final de los adjuntos, en concordancia con la función sintáctica del participante acerca del cual predican. Este rasgo tipológicamente sobresaliente, llamado "concordancia del participante" (CP), opera tanto al interior de la cláusula como en la combinación de cláusulas. En este último ámbito sintáctico los marcadores de $\mathrm{CP}$ forman parte del sistema de cambio de referencia (switch-reference). El presente artículo trata la $\mathrm{CP}$ en lenguas pertenecientes a diferentes subconjuntos de la familia Pano y propone que este rasgo formaba parte de la gramática Proto-Pano. La discusión sobre el posible desarrollo diacrónico de este sistema conduce a una hipótesis acerca de la existencia de un sistema de marcación de caso tripartito en la proto-lengua.
\end{abstract}

Palabras clave: Familia Pano. Adjuntos. Concordancia del participante. Proto-Pano. Marcación de caso. Cambio de referencia (switch-reference).

\section{Resumo}

Nas línguas da família Pano, uma série de construções adjuntas (que expressam lugar, etapa da vida, quantificação distributiva, maneira, entre outros) está semanticamente orientada para um dos participantes obrigatórios da cláusula. Tal relação é marcada explicitamente mediante uma morfologia reflexiva ao final dos adjuntos, em concordância com a função sintática do participante sobre o qual predica. Este traço tipologicamente sobressalente, chamado "concordância do participante" $(\mathrm{CP})$, opera tanto no interior da cláusula quanto na combinação das cláusulas. Nesse último âmbito sintático, os marcadores de $\mathrm{CP}$ fazem

1 Deseo expresar mi sincero agradecimiento al Laboratorio de Lenguas Indígenas (LALI) de la Universidad de Brasilia, y en especial a los profesores Aryon Rodrigues y Ana Suelly, por su generosa invitación a participar del Encuentro Internacional "Arqueología y Lingüística Histórica de las Lenguas Indígenas de Sudamérica”.

Este artículo es una versión revisada de Valenzuela (2003, capítulos 19 y 20) y echa mano de los avances alcanzados en los estudios lingüísticos Pano durante la última década, en particular en lo referente a la descripción de sus sistemas de marcación de caso y de concordancia del participante.

2 Chapman University. 
parte do sistema de referência alternada (switch-reference). O presente artigo trata da CP nas línguas pertencentes a diferentes subconjuntos da família Pano e propõe que esse traço fazia parte da gramática do Proto-Pano. A discussão sobre o possível desenvolvimento diacrônico desse sistema conduz a uma hipótese acerca da existência de um sistema de marcação de caso tripartido na proto-língua.

Palavras-chave: Família Pano. Adjuntos. Concordância do participante. Proto-Pano. Marcação de caso. Referência alternada (switch-reference).

\section{Introducción}

\subsection{El sistema de concordancia del participante}

En las lenguas de la familia Pano, una serie de construcciones adjuntas (que expresan lugar, manera, cuantificación distributiva, entre otros) suelen predicar acerca de uno de los participantes obligatorios de la cláusula y no acerca del evento en su totalidad. Dicha orientación semántica es marcada en los adjuntos mediante una morfología flexiva que concuerda con la función sintáctica del participante acerca del cual predican. Este rasgo, conocido como "concordancia del participante" (en adelante, CP), es probablemente la característica tipológica más sobresaliente de la gramática Pano (Valenzuela 2003, 2005a). A manera de ilustración, considérense las siguientes oraciones de la lengua chákobo (Valenzuela 2005b)3:

(1) sobo napatá ina hirí paßi-ma-ki. casa sobre.el.piso:O perro:ABS Jere:ERG bailar-CAUS-COMPL 'Jere hizo bailar al perro sobre el piso de la casa' ${ }^{4}$. (ubicación del perro)

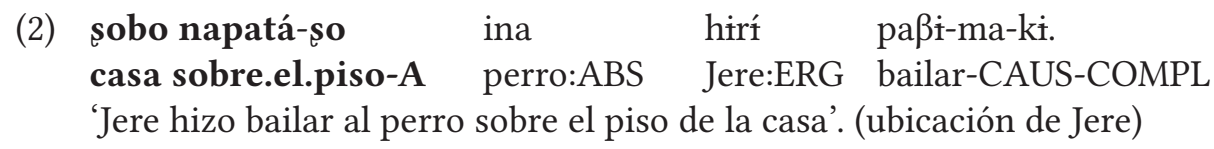

A diferencia de su traducción española, las oraciones (1) y (2) no son ambiguas en chákobo. La expresión locativa sobo napatá 'sobre el piso de la casa' ocurre por sí sola cuando predica acerca del participante en función de $\mathrm{O}$ (objeto), pero lleva el morfema adicional -so cuando predica acerca del participante en función de A (sujeto transitivo). Es decir, (1) indica que el perro está sobre el piso de la casa (y no al ras del suelo) cuando el evento tiene lugar,

3 A lo largo de este trabajo se emplearán los símbolos S, A y O siguiendo a Dixon (1994). $\mathrm{S}$ se refiere al sujeto de un verbo intransitivo, en tanto que $\mathrm{A}$ y $\mathrm{O}$ se refieren al sujeto y al objeto de un verbo transitivo, respectivamente.

4 Las viviendas chákobo generalmente son sostenidas por palos gruesos prendidos en la tierra, de manera que el piso de la casa y el suelo están en diferentes niveles. Ejemplos equivalentes a (1) y (2) fueron inicialmente discutidos en un estudio acerca de la causativización en la lengua hermana shipibo-konibo (Valenzuela 2002:467-468). 
en tanto que Jere no comparte esta ubicación (probablemente esté parado al ras del suelo); por el contrario, (2) indica que Jere está sobre el piso de la casa mientras que el perro puede compartir esta ubicación o no. Por lo tanto, expresiones adjuntas de lugar tales como sobo napatá(-so) indican la ubicación de uno de los participantes y no del evento en su totalidad.

En la oración (3), también perteneciente a la lengua chákobo, encontramos una expresión locativa marcada por - $2 a s$ y controlada por el participante en función de $\mathrm{S}$ o sujeto intransitivo:
a. hiwi-?as
$\dot{\mathrm{i}}$
pakí-ki.
árbol-de:S 1:NOM caer-COMPL
'Me caí del árbol'.

Una particularidad del idioma chákobo es que los adjuntos presentan una forma larga cuando ocurren en posición postverbal (ver también la oración (6)):
b. $\dot{\mathrm{i}}$ pakị-ki hiwi-?as-na. 1:NOM caer-COMPL árbol-de:S-forma.larga 'Me caí del árbol'.

Como resulta evidente al examinar las oraciones presentadas arriba, la $\mathrm{CP}$ está relacionada a la transitividad en el sentido de que la orientación hacia el participante en función de A (ejemplo (2)) se marca de manera distinta a la orientación hacia el participante en función de S (ejemplo (3)). Sin embargo, la transitividad no es el factor determinante en la distribución de los marcadores puesto que las cláusulas (1) y (2) tienen, obviamente, el mismo valor transitivo pero exhiben morfemas de $\mathrm{CP}$ distintos dependiendo de si la expresión locativa está orientada hacia el participante $\mathrm{O}$ o el participante $\mathrm{A}$, respectivamente (ver también la oración (12)) $)^{5}$.

El objetivo de este trabajo es ofrecer un análisis de la $\mathrm{CP}$ en las lenguas Pano desde una perspectiva comparativa y proponer un escenario histórico del proceso a través del cual se habría desarrollo dicho sistema. De esta manera se busca contribuir a la comprensión del que considero el rasgo más destacado de la gramática Pano y avanzar nuestro conocimiento de la protolengua. Pero antes de abordar este tema, en lo que resta de la sección 1 se hace una breve presentación de la familia lingüística Pano, los principales rasgos gramaticales compartidos por las diferentes lenguas y las lenguas consideradas en el presente estudio. Posteriormente, en la sección 2 se analiza la CP tanto a nivel de la combinación de cláusulas como al interior de la cláusula. La sección

5 Por lo tanto, no es acertado referirse a este fenómeno con los nombres de "concordancia de la transitividad" o "concordancia transitiva" (véase, por ejemplo, Loos 1999, Valenzuela 1999, Fleck 2010, entre otros). 
3 discute el fenómeno analizado desde una perspectiva interlingüística. Se establece una similitud entre la $\mathrm{CP}$ y los predicados secundarios descriptivos en otras lenguas del mundo, haciendo alusión especial a construcciones que exhiben concordancia de caso en los adjuntos en algunas lenguas aborígenes de Australia. Sin embargo, la CP también presenta peculiaridades que se explican mediante su particular desarrollo diacrónico y la hacen un fenómeno único ${ }^{6}$. La sección 4 examina las implicancias del análisis en la sección 2 para la gramática del Proto-Pano y propone una reconstrucción de los morfemas de $\mathrm{CP}$ así como del posible escenario histórico mediante el cual este sistema se habría desarrollado. Finalmente, la sección 5 presenta los comentarios finales.

\subsection{La familia lingüística Pano}

La familia lingüística Pano es una bien establecida entidad genética de las tierras bajas de Sudamérica. Comprende una treintena de lenguas (que solían ser) habladas en los bosques tropicales adyacentes de las actuales repúblicas del Perú (regiones Loreto, Ucayali, Huánuco y Madre de Dios), Brasil (estados Amazonas, Acre, Rondonia) y Bolivia (departamentos Beni y Pando).

La homogeneidad de las lenguas Pano fue reconocida muy tempranamente por los misioneros europeos según se puede comprobar, por ejemplo, en el relato del padre Figueroa, quien en 1661 escribe: "Algunas [lenguas] se han hallado que son algo comunes... La de los barbudos, con los chipeos, cheteos y capanaguas ${ }^{7 ”}$ (1904[1661]:187). En 1888 Raoul de la Grasserie establece formalmente la existencia de la familia lingüística Pano entre la comunidad académica, mediante un trabajo presentado ante el VII Congreso Internacional de Americanistas realizado en Berlin (de la Grasserie 1890). Este autor analiza listas léxicas de las que llamó siete "lenguas" Pano (se mantiene los nombres y ortografía tal como en el original): Pano, Conibo, Pacavara, Caripuna, Culino, Maxuruna, Mayoruna Domestica y Mayoruna Fera. Tras presentar correspondencias fonéticas regulares, concluye que estas forman parte de una misma unidad genética y adopta el nombre "Pano" para referirse a la familia lingüísticå. "Pano" significa "armadillo gigante" (Priodontes maximus) (Tessmann 1999:58).

\footnotetext{
6 Por ejemplo, Valenzuela (2003:83-84) sugiere que dado que los marcadores de CP están asociados diacrónicamente a la concordancia de caso y presentan una configuración general tripartita, este rasgo podría ser visto como un tipo especial de escisión de la ergatividad exclusivo de las lenguas Pano.

7 Es decir, mayoruna o matsés, shipibos, shetebos y kapanawas.

8 De la Grasserie también incluye comentarios gramaticales acerca de una de las lenguas llamada pano, tomados al parecer de Castelnau (1851:292-293). Esta lengua es mejor conocida actualmente como wariapano/huariapano, y podría tratarse de una variedad de shetebo (Erikson 1999:44).
} 
La población Pano puede ser estimada en unos 50.000 individuos (Córdoba et alia, 2012). Una característica a resaltar es la existencia de una notable homogeneidad cultural, lingüística y territorial (a excepción de la zona BoliviaRondonia) inusual para una población relativamente grande, que contrasta con el mantenimiento de un número impresionante de unidades sociológicas autónomas (Erkison et alia 1994:4). Además, los pueblos Pano exhiben diferencias significativas en cuanto a su demografía, tiempo e intensidad del contacto con las sociedades envolventes, grado de vitalidad de la lengua propia y bilingüismo en español o portugués (Valenzuela 2003). También se atribuye a estos pueblos una conciencia de pertenecer a un conjunto Pano supralocal (concebido a partir de sus vecindades) con contornos muy difusos. Así, los sharanawa consideran que los pueblos Pano vecinos comparten el mismo origen mitológico, en tanto que los kashinawa reconocen que el empleo del sistema de "nombres verdaderos" define a la "gente de homonimia". Estudiosos como d'Ans (1970:13) y Loos (1999:227) han hecho mención de la semejanza estructural de las lenguas Pano. Según Erikson (1999:52), esta homogeneidad lingüística "es tanto efecto como causa de su cohesión". Así, se habla de una voluntad política de mantener la intercomprensibilidad mutua, lo cual jugaría un papel esencialmente centrípeto (Erikson, op. cit.). Muestras de esto último serían el uso de un pidgin Pano en la zona del Alto Purús (Shell 1975:25, Loos 1999:228), la adopción concertada entre algunos shipibo de rasgos fonológicos de aldeas vecinas a fin de evitar diferenciarse demasiado lingüísticamente (Levy 1991:5) y la insistencia de los matís respecto a la identidad de su habla con aquellas de sus vecinos korubo y matsés (Erikson 1999:52).

\subsection{Rasgos gramaticales de las lenguas Pano}

Entre los rasgos gramaticales que caracterizan a los idiomas Pano cabe destacar los siguientes (ver también Valenzuela 2003:882):

a. Orden básico de constituyentes AOV/SV

b. Morfología predominantemente aglutinante con tendencia polisintética en el verbo

c. Uso casi exclusivo de sufijos y posposiciones

d. Prefijos de partes del cuerpo que se combinan con nombres, adjetivos y verbos, y que suelen cumplir una función locativa

e. Supleción en verbos de movimiento en base a la transitividad: "ir"/"llevar", "venir"/"traer"

9 Esta actitud contrasta con lo reportado acerca de pueblos pertenecientes a otras familias de dimensiones comparables como los Tukano, para quienes las diferencias lingüísticas constituyen emblemas de identidad (Erikson 1999:51-53; Sorensen 1967). 
Mapa 1. Distribución de las Lenguas Pano (Erikson 1999)

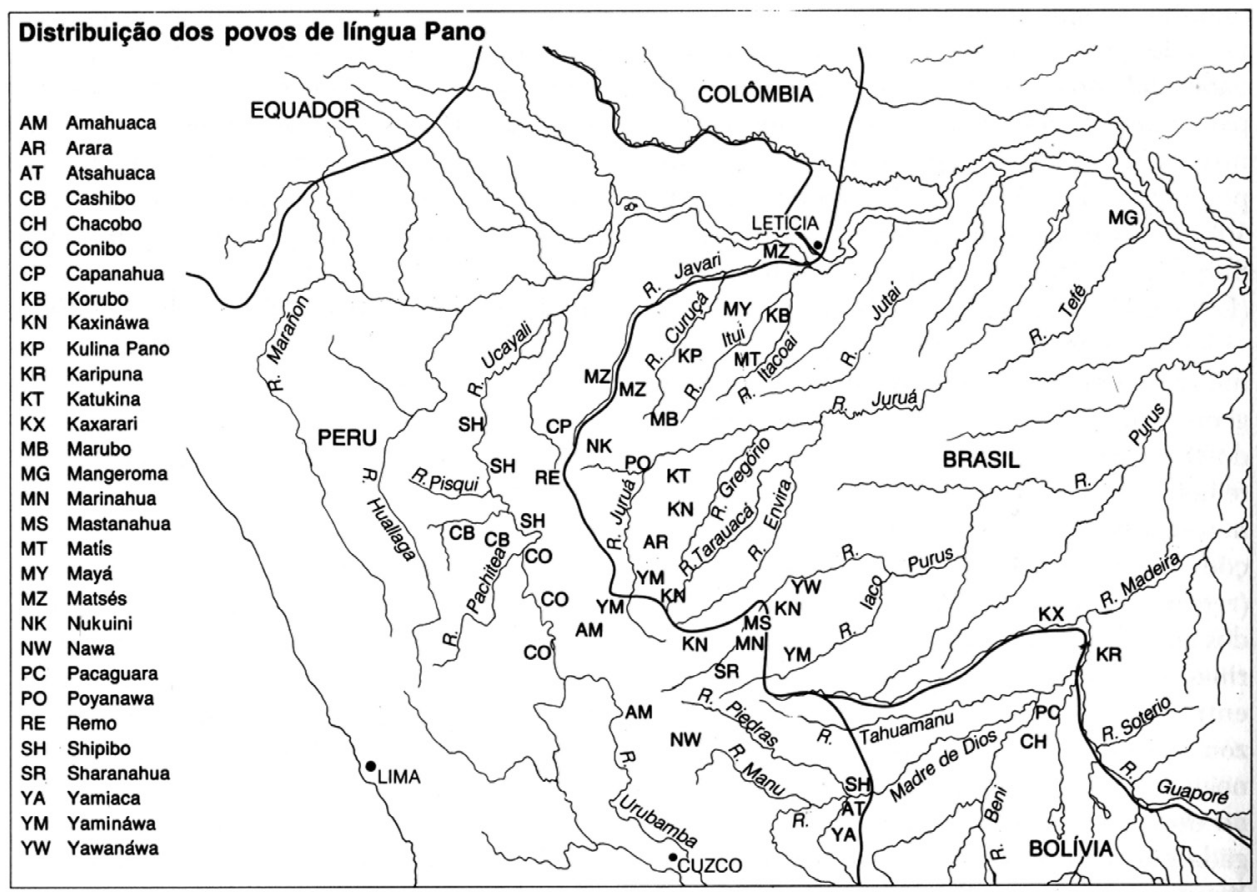

f. Ausencia (o desarrollo incipiente) de marcadores de persona (cognados) en el verbo principal o auxiliar. Algunas lenguas distinguen entre los participantes del acto de habla y la tercera persona

g. Sistemas de marcación de caso ergativo con diversos tipos de escisiones

h. Sincretismo o polifuncionalidad de las formas ergativa, instrumental, genitiva, locativo-direccional y otras formas oblicuas ${ }^{10}$

i. Ocurrencia de los marcadores de caso al final del sintagma nominal

j. Ausencia de cualquier tipo de concordancia al interior del sintagma nominal

k. Distinción estricta entre verbos intransitivos y transitivos

1. Presencia de dos auxiliares o pro-verbos: el intransitivo contiene la $\mathrm{vocal} / \mathrm{i} /$ mientras que el transitivo contiene la vocal /a/

m. Concatenación de cláusulas (clause chaining) y sistemas complejos de cambio de referencia (switch-reference) con morfología cognada

n. Concordancia del participante $(\mathrm{CP})$ en ciertos adjuntos

10 El sincretismo de una función puede ser parcial como ocurre con el locativo en shipibokonibo, que exibe una forma idéntica al ergativo (incluyendo sus varios alomorfos), pero también los morfemas alternativos -no, -nko y -ain. 
o. Casos de armonía transitiva en la frase verbal expresada en sufijos verbales, verbos auxiliares y verbos de fase

p. Empleo de clíticos pronominales duplicados ("clitic doubling") en ciertas lenguas

q. Sistemas complejos de morfemas verbales de movimiento

r. Sistemas temporales métricos que distinguen diferentes grados de distancia a partir del centro deíctico

s. Sistemas evidenciales con morfología no cognada (con la posible excepción del reportativo) a través de las distintas agrupaciones de lenguas

En este trabajo se demuestra que la $\mathrm{CP}$ (rasgo n en la lista arriba) está presente en todas las subdivisiones de la familia $P_{a n o}{ }^{11}$ y se manifiesta en dos niveles sintácticos. Además, el estudio comparativo de este rasgo tiene consecuencias importantes para nuestro conocimiento de la gramática del Proto-Pano.

\subsection{Selección de lenguas en el presente estudio}

Existen diferentes clasificaciones internas de la familia Pano, entre ellas el trabajo pionero de Shell (1975) mediante la aplicación del método comparativo, Loos (1999) basado en características compartidas de nivel fonológico, morfológico y en menor grado léxico, Amarante Ribeiro (2005) empleando métodos cladísticos (Fabre 2005) y Fleck en base a la comparación léxica y gramatical (de pronta publicación). Posiblemente la postura más conservadora (en el sentido que se explicitará abajo) es la que encontramos en Erikson (1999:48-51), quien reporta basarse en criterios "esencialmente lingüísticos" y reconoce ocho "subconjuntos principales":

1. Shipibo-konibo

(Erikson 1999:48) los considera "los más divergentes de los grupos panos")

2. Panos meridionales: chákobo, pakawara, karipuna, kaxararí1 ${ }^{12}$

3. Yaminawas: "Panos del Purús": yaminawa, sharanawa, mastanawa, marinawa, morunawa, yora

4. Amawaka

11 Carecemos de datos suficientes acerca de la lengua kaxararí (aunque Erikson (1999) lo incluye dentro del Pano meridional (ver abajo), Lewis (2009) lo lista como un subgrupo independiente). Sin embargo, los pocos datos kaxararí a los que hemos podido acceder sugieren procesos de simplificación en su sistema de CP (Valenzuela y Oliveira, notas de campo, 2012). Carecemos también de información suficiente sobre idiomas extintos como el atsawaka y yamiaka que parecen haber conformado un grupo diferenciado (Shell 1975:111).

12 El idioma kaxararí es ubicado en un subgrupo independiente según la clasificación en Lewis (2009). 
5. Kashinawa

6. Kashibo(-kakataibo)

7. Panos Medianeros: kapanawa, marubo, katukina, poyanawa, remo

8. Mayorunas o panos septentrionales: matsés, matís, korubo

Los subconjuntos presentados por Erikson con compatibles con las "ramas" postuladas en Valenzuela (2003), con dos excepciones: Valenzuela no distingue yaminawa de kashinawa, ni tampoco shipibo-konibo de los llamados "panos medianeros" (Erikson considera a este último grupo como el "eslabón perdido" entre el shipibo-konibo y los panos septentrionales). Ambos autores coinciden en un punto importante (también destacado en Amarante Ribeiro, 2005): la necesidad de tratar al amawaka separadamente del yaminawa y kashinawa. En este estudio se considera al menos una lengua de cada uno de los ocho subconjuntos propuestos por Erikson, adoptando así una actitud conservadora, en el sentido de ser muy probablemente sobre-diferenciadora.

El Cuadro 1 a continuación resume información básica con respecto al número aproximado de hablantes y la ubicación general de las lenguas seleccionadas. A menos que se consigne otra fuente, la información proviene de Lewis (2009).

Cuadro 1. Lenguas Incluidas en el Estudio

\begin{tabular}{|l|l|l|}
\hline subconjunto & Lengua & $\begin{array}{l}\text { ubicación /número aproximado de hablantes } \\
\text { tomado de Lewis (2009) a menos que se } \\
\text { indique una fuente distinta (véanse las } \\
\text { referencias en Lewis) }\end{array}$ \\
\hline sureño & Chákobo & $\begin{array}{l}\text { Bolivia, Beni: 1,000. } \\
1 \text { familia entre los chákobo. } \\
\text { (Córdoba et alia 2012) }\end{array}$ \\
\hline purús & Yaminawa & $\begin{array}{l}\text { Perú, Ucayali y Madre de Dios: } 750 \text { (2003). } \\
\text { Brasil, Acre: 500. } \\
\text { Bolivia, Pando: 140. } \\
\text { Población total: 1,390 (incluye chitonawa y } \\
\text { mastanawa). }\end{array}$ \\
\hline amawaka & Amawaka & $\begin{array}{l}\text { Perú, Ucayali y Madre de Dios: 110 (2000). } \\
\text { Brasil, Amazonas: 220 (1995). } \\
\text { Población total en ambos países: 330. Población } \\
\text { total del grupo étnico: 500. } \\
\text { Tal vez 50 no contactados en frontera Perú-Brasil. }\end{array}$ \\
\hline kashinawa & Kashinawa & $\begin{array}{l}\text { Perú, Ríos Curanja y Purús en Ucayali: 1,600 (2003). } \\
\text { Brasil, Acre: 400 (2003). } \\
\text { Población total en ambos países: 2,000. } \\
\text { Población total grupo étnico: 5,000 }\end{array}$ \\
\hline
\end{tabular}




\begin{tabular}{|l|l|l|}
\hline ucayalino & $\begin{array}{l}\text { shipibo- } \\
\text { konibo }\end{array}$ & $\begin{array}{l}\text { Perú, Ucayali, Loreto, Huánuco, Madre de Dios: } \\
\text { 30,000-40,000 (Valenzuela 2003). }\end{array}$ \\
\hline medianero & kapanawa & Perú: Ríos Tapiche y Buncuya, Loreto. 390 (2000). \\
\hline occidental & $\begin{array}{l}\text { kashibo- } \\
\text { kakataibo }\end{array}$ & $\begin{array}{l}\text { Perú, Ucayali y Huánuco: 1,879 (INEI 2007), 3,500 } \\
\text { (Zariquiey 2011). }\end{array}$ \\
\hline norteño & Matsés & $\begin{array}{l}\text { Perú, Loreto: 1,400 (2006). } \\
\text { Brasil, Amazonas: } 800(2006) . \\
\text { Brasil, Amazonas: } 240(2000) .\end{array}$ \\
\hline
\end{tabular}

En la presentación de los datos se emplea una trascripción uniformizada cercana al nivel fonológico, empleando en lo posible símbolos del Alfabeto Fonético Internacional. Las fuentes de las cuales han sido extraídos los ejemplos son indicadas en cada instancia. Se han mantenido las glosas originales a excepción de las correspondientes a la $\mathrm{CP}$, las cuales han sido regularizadas. Lamentablemente, algunas fuentes no proporcionan un análisis morfémico de los datos. En estos casos, se ofrecen glosas provisionales basadas en el análisis de la información gramatical en la misma fuente u otra fuente adicional. Esto, sin embargo, no afecta lo esencial de los puntos bajo discusión ${ }^{13}$.

\section{Concordancia del participante en las lenguas Pano}

En las lenguas Pano la $\mathrm{CP}$ se manifiesta en dos niveles sintácticos: al interior de la cláusula y en la combinación de cláusulas. En adelante me referiré a estos dos niveles como "intra-oracional" e "inter-oracional", respectivamente ${ }^{14}$.

13 Los símbolos y abreviaturas empleados a lo largo del artículo son los siguientes: 1 primera persona, 2 segunda persona, 3 tercera persona, A (orientación semántica hacia el) sujeto transitivo, ABL ablativo, ABS absolutivo, ACU acusativo, ADVZR adverbializador, AGT agente, APROX aproximado, AUM aumentativo, AUX auxiliar, COM comitativo, COMPL completivo, CONJ conjunción, C.R. cambio de referencia, DECL declarativo, DIM diminutivo, DIR direccional, DIST distante, ENF enfático, ERG ergativo, EV evidencial directo, GEN genitivo, HAB habitual, IMPF imperfectivo, INCOMPL incompletivo, INDIC indicativo, INF infinitivo, INSTR instrumental, INT interrogativo, INTR intransitivo, LOC locativo, NAR narrativo, NEG negativo, NOM nominativo, NMLZR nominalizador, O (orientación semántica hacia el) objeto, PAC paciente, PASD pasado, PERF perfectivo, PL plural, POST posterior, PRES presente, PREV previo, PROGR progresivo, $\mathrm{PROP}$ propietivo, PROX próximo, PRTCL particular, PRTCP participio, REC reciente, REM remoto, REP reportativo, REPE repetitivo, S (orientación semántica hacia el) sujeto intransitivo, SD sujetos diferentes, SEC secuencial, SG singular, SI sujetos idénticos, SIM simultáneo, SUJ sujeto, TEMP temporal, TR transitivo, VNC vinculador.

14 Hasta hace relativamente poco tiempo el análisis del sistema de CP se había visto obstaculizado por la tendencia de los lingüistas de tratar sus manifestaciones en estos dos niveles como fenómenos distintos, o por enfocarse de manera exclusiva en el nivel interoracional al tratar de describir el sistema de cambio de referencia (switch-reference). 


\subsection{Concordancia del participante a nivel inter-oracional}

Como se anticipó en 1.3, las lenguas Pano poseen sistemas complejos de cambio de referencia. Esto permite combinar dos o más cláusulas en una misma cadena de habla, sin necesidad de expresar en cada cláusula la identidad de los argumentos mediante sintagmas nominales (recuérdese que las marcas de sujeto y objeto son prácticamente inexistentes o incipientes en el verbo Pano). Estas construcciones complejas no resultan ambiguas para los hablantes en gran medida debido a que los morfemas de $\mathrm{CP}$ sirven como marcadores de sujetos idénticos, es decir su presencia/ausencia indica si los sujetos de dos cláusulas se refieren al mismo participante o $\mathrm{no}^{15}$. Asimismo, estos marcadores establecen el orden temporal-lógico de los eventos expresados en las cláusulas que enlazan. Sin embargo, podemos decir que su función básica es la de orientar la cláusula marcada hacia el participante S ó A de la cláusula matriz. El Cuadro 2 presenta los marcadores de (no)correferencia a nivel inter-oracional. En negrita resaltamos aquellos que indican CP.

Las correspondencias fonológicas relevantes entre el Proto-Pano ${ }^{16}$ y las lenguas hijas son las siguientes:

- *s tiene los reflejos /s/ en chákobo, kapanawa, yaminawa, kashinawa, shipibo-konibo y kashibo-kakataibo, /x/ en Amawaka y / / en matsés.

- Una característica de la lengua chákobo es la pérdida de $/ \mathrm{n} /$ en posición de coda en los marcadores controlados por el participante en función de A (i.e., las columnas 2 y 4) (Valenzuela e Iggesen 2007).

- En cuanto a las vocales, ${ }^{*} \mathrm{i}$ exhibe los reflejos /i/ y /e/ en matsés, y *o tiene los reflejos /o/ y /u/ en matsés y kashibo-kakataibo.

Al observar el Cuadro 2 podemos generalizar que los marcadores que indican concordancia con el participante en función de A terminan en $/ \mathrm{n} / \mathrm{o}$ nasalización. La excepción son las lenguas sureñas, en este caso el chákobo, conforme a la innovación fonológica mencionada arriba. En contraste, los marcadores que indican concordancia con $S$ terminan en /s/ o /i/, o sus equivalentes.

15 Además de marcadores de sujeto idéntico y de sujeto diferente, las lenguas Pano suelen poseer morfemas que expresan la identidad entre objeto y sujeto intransitivo/transitivo (como se muestra en la última columna del Cuadro 2). Aunque por lo general solo los marcadores de sujeto idéntico exhiben marcación de $\mathrm{CP}$, existen excepciones como $-k t_{i}$ vs. -kişun en kashibo-kakataibo (Cuadro 2), véase también el marcador amawaka -xo ilustrado en la oración (12).

16 Se considera que en estos casos la reconstrucción fonológica ofrecida por Shell (1975) para el Pano-Reconstruido es válida para el Proto-Pano. 


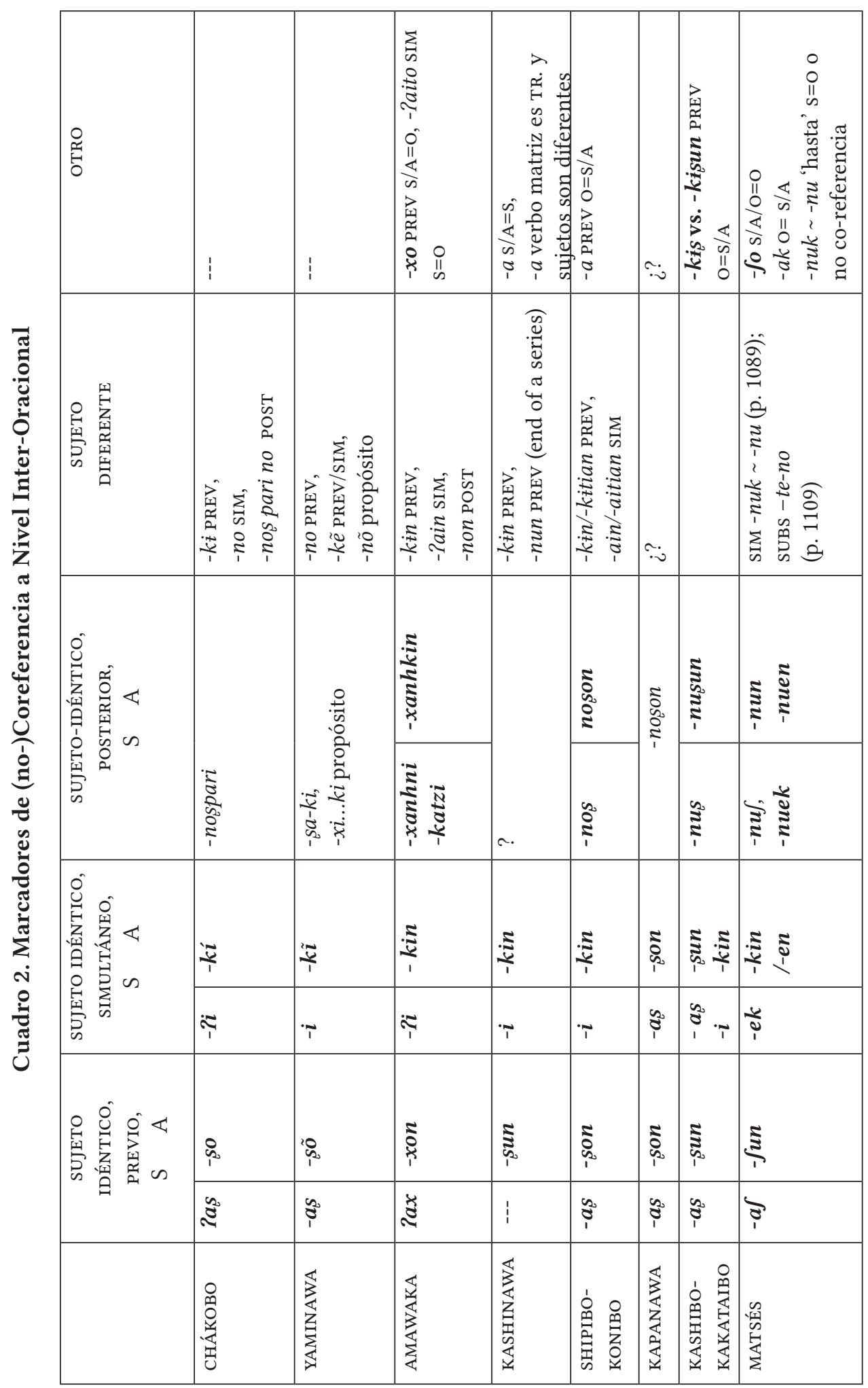




\subsubsection{Evento de la cláusula dependiente precede al evento de la cláusula matriz}

En el ejemplo (4) encontramos una cláusula dependiente marcada por - as (que aparece entre corchetes) y una cláusula finita intransitiva. El morfema - as indica que el evento descrito por la cláusula dependiente (quemar la chacra) es previo al evento de la cláusula matriz (ir al monte) (PREV), los sujetos son idénticos (SI) y la cláusula dependiente predica acerca del participante $S$ de la cláusula matriz $(\mathrm{S})$ :

\section{SHIPIBO-KONIBO}
(4) nokon papa-ra [wai mino-as]
POS1 padre:ABS-EV chacra:ABS quemar-PREV.SI.S

$\begin{array}{ll}\text { katfio } & \text { ka-ki. } \\ \text { al.monte } & \text { ir-COMPL }\end{array}$
'Mi padre, quemando la chacra, se fue al monte'.
'Después de quemar la chacra, mi padre se fue al monte'.

A diferencia de (4), la cláusula matriz en (5) es transitiva (sembrar yuca). Por lo tanto, se requiere del morfema -son en la cláusula dependiente para indicar que esta predica acerca del participante A de la cláusula matriz:

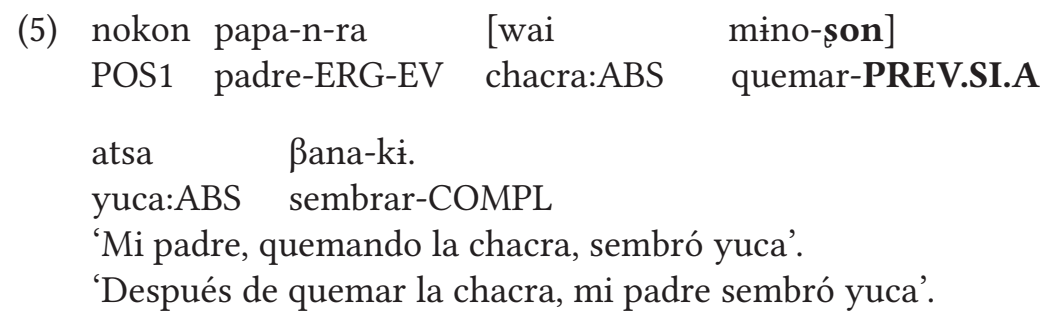

Como resulta evidente, a nivel inter-oracional la concordancia con el participante $S$ de la cláusula matriz se correlaciona con la presencia de un verbo intransitivo en dicha cláusula, mientras que la concordancia con el participante A se correlaciona con la presencia de un verbo transitivo.

CHÁKOBO (Zingg 1998, Valenzuela 2005b)

El chákobo es peculiar ya que los marcadores de sujetos idénticos (es decir, $\mathrm{CP}$ inter-oracional) exhiben formas cortas o formas largas (FL) dependiendo de la posición de la cláusula dependiente o marcada en la oración. La versión larga se atestigua cuando la cláusula marcada ocurre al final de la oración (recuérdese también las formas alternativas de la expresión locativa en el ejemplo (3)): 
(6) a. [paki-Tas] tsi ha ará-ki. caer-PREV.SI.S VNC 3:NOM llorar-COMPL

b. ha ará-ki [paki-?as-na].

3:NOM llorar-COMPL caer-PREV.SI.S.FL

'El lloró porque se había caído’. (Zingg 1998:32) / 'Cayéndose, él lloró'.

Recuérdese que en esta lengua los marcadores que indican concordancia con A pierden la /n/ en posición de coda:

(7) [wai-?aş ho-so] josá hawí ßakí chaco:ABL-S venir-PREV.SI.A mujer:ERG 3:GEN niño:ABS ía $\quad \beta i-k i ́$.

piojo:ABS conseguir-COMPL

'Después de regresar del chaco, la mujer despiojó a su hijo' (Valenzuela 2005b:193).

YAMINAWA (Faust y Loos 2002:149)

(8) [mã pi-as $]$ nõ maikiri ka-ta-ita. ya comer-PREV.SI.S nosotros río.abajo ir-DIST-ayer 'Habiendo comido, fuimos río abajo'.

(9) [soo mirã tsao-sõ], ẽ koma oĩ-ta. escondite dentro sentarse-PREV.SI.A 1 SG perdiz ver-ayer 'Habiéndome sentado en el escondite, vi una perdiz ayer'.

AMAWAKA (Sparing-Chávez 1998)

Recuérdese que /x/ es el reflejo de *s en amawaka:

(10) [jantan Poro-?ax-min] tarde cultivar-PREV.SI.S-TEMA

hatzanami-i-yama-shin-ki-?in. cansarse-REFL-NEG-1-3.días.atrás-1PASD-DECL 'A pesar de que cultivé hasta tarde ayer, no me cansé'. (p. 457)

(11) [ni?i ka-xon-min] ?in ?aa riti-ki-?in. monte ir-PREV.SI.A-TEMA yo tapir matar-1PASD-DECL 'Fui al monte y maté un tapir'. (p. 458)

Una particularidad del amawaka es que la cláusula dependiente puede estar orientada hacia el objeto de la cláusula matriz. Esta relación se codifica mediante un morfema que indica correferencialidad del sujeto de la cláusula 
dependiente con el objeto de la cláusula matriz. De manera crucial, el marcador empleado en este caso es - $\boldsymbol{x} \boldsymbol{o}$ (es decir, $\sin / \mathrm{n}$ / final o nasalización):

(12) koriiki $\beta$ i-xo-min

han ?ija ?atji-xo-?in.

dinero tomar-PREV.S/A=O-TEMA él a.mí agarrar-3PASD-DECL

'Después de que yo tomé el dinero, él me agarró'. (p. 458)

Nótese que la alternancia entre -xon y -xo en los ejemplos (11) y (12) no puede ser explicada en base a la transitividad del verbo en la cláusula matriz, puesto que en ambos casos se trata de un verbo transitivo. En conclusión, los marcadores de $\mathrm{CP}$ inter-oracional amawaka presentan la siguiente distribución tripartita según la orientación semántica de la cláusula marcada con uno de los participantes de la cláusula matriz: - Pax $=$ concordancia con $\mathrm{S},-x o n=$ concordancia con A, -xo concordancia con O. Es más, Sparing-Chávez (op. cit.) considera que estos marcadores son analizables de la siguiente manera:

$\begin{array}{lll}-\mathrm{Pa} & +-\mathrm{x} & \text { nominativo } \\ -\mathrm{xo} & +-\mathrm{n} & \text { ergativo } \\ -\mathrm{xo} & +-0 & \text { absolutivo }\end{array}$

\section{KASHINAWA}

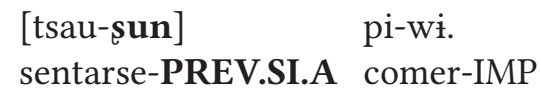

'Siéntate y come'. (Montag, 2008:58)

(14) $[\text { Bai anu ka-tan-sun }]^{17}$

chacra ahí ir-hacer.y.volver-PREV.SI.A

nukun ainin nuku $\quad \beta a \beta a-s ̧ n-m i s-k i$.

nuestras esposas nosotros cocinar-BEN-HAB-DECL

'Después de ir y volver de la chacra, nuestras esposas siempre cocinan para nosotros'. (R. Montag 2005:6)

Según Montag (2008:58), en kashinawa el sufijo - a tiene dos usos. El primero corresponde al del morfema -(2)as en las lenguas hermanas; es decir, el verbo matriz es intransitivo y los sujetos de las dos cláusulas son los mismos.

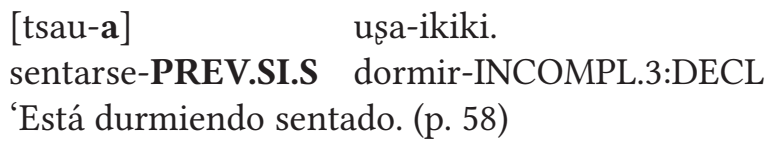

17 Aunque en este caso el autor emplea el morfema - shun (o sea, - $\int u n$ ), en una descripción posterior propone la forma-sun (es decir, con una consonante sibilante retrofleja). Vale la pena notar que en kashinawa el sufijo -su es un marcador de pasado del mismo día (Montag 2008:60-61). 
Construcciones como la ofrecida en (15) sugieren que en kashinawa el morfema - as devino en - $a$, es decir que se produjo una pérdida de la consonante retrofleja sibilante en posición de coda (véase también el ejemplo ).

KASHIBO-KAKATAIBO (Zariquiezy 2011)

En kashibo-kakataibo, -as y -sun se emplean no solo cuando el evento de la cláusula dependiente es previo al de la cláusula matriz, sino también cuando ambos eventos son simultáneos (además, estos marcadores pueden tener una interpretación condicional).

$\begin{array}{lll}\text { [[pi-as] } & \text { kana] } & \text { ?abat-i-n } \\ \text { comer-PREV/SIM.SI.S } & \text { NAR.1SG } & \text { correr-IMPF-1/2 }\end{array}$

'Después de comer, corrí' (p. 576).

$\begin{array}{lll}\text { [[pi-sun] } & \text { kana] } & \text { sia-i-n } \\ \text { comer-PREV/SIM.SI.A } & \text { NAR.1SG } & \text { beber-PERF-1/2 }\end{array}$

'Después de comer bebí'. (p. 578)

En kashibo-kakataibo existe otro par de morfemas, -tankis y -tankisun, especializados en marcar evento previo y concordancia con S o A, respectivamente. Nótese que tanto -as como -tankis exhiben la terminación $-s$, mientras que -sun y -tankișun exhiben la terminación -(su)-n. Por otro lado, las oraciones y presentan los marcadores de sujetos idénticos empleados exclusivamente cuando los eventos son simultáneos.

KAPANAWA (Loos 1999:238)

(18) [mai kintí ki kobin-?a $\quad \beta i \quad$ ki siniska-as] barro olla en cocinar-C.RperoREP envejecerse-PREV.SI.S

$\beta i$ imiki?t-i.

romperse-PRES

'Cuando se cocina en olla de barro vieja (que se ha envejecido), ésta se rompe'.

(19) [nonti ?aniha-son] miin pitißo-ri?ßi $\quad \beta a n a w i$ canoa agrandar-PREV.SI.A tu comida.también planta hano napo-ja?-noson. ahí amontonar-FUT-SUBS.SI.A

'Tras agrandar tu canoa, planta tus alimentos también para amontonarlos ahí'.

MATSÉS (Fleck 2003)

Recuérdese que en matsés / $/$ / es el reflejo de *s. 
adenbidi opa tsine-sun mikweste pe-kid. también:TR perro cazar.con-PREV.SI.A añuje comer-HAB 'También los matsés cazan añuje (agutí) con perro y los comen’. (p. 1096)

(21) podked-wa-af kapu-kid camino-hacer-PREV.SI.S deambular-NMLZR.AGT

tanbis ne-e-k. majaz ser-no.PASD-INDIC

'Los majaces (pacas) deambulan después de hacer camino' (p. 1096)

'Pacas are ones that walk around after making paths.'

Similarmente al amawaka, el idioma matsés tiene un marcador de forma - - o que indica orientación de la cláusula dependiente hacia el objeto de la cláusula matriz. Específicamente, este morfema indica correferencia entre un participante de la cláusula dependiente $(\mathrm{S} / \mathrm{A} / \mathrm{O})$ y el objeto de la cláusula matriz.

\subsubsection{Eventos Simultáneos}

Cuando los eventos de las dos cláusulas son (parcialmente) simultáneos, las diferentes lenguas Pano echan mano de los marcadores - kin kĩ , para indicar concordancia con el participante en función de A en la cláusula matriz, o - $i i ~ ~-i$, cuando la cláusula marcada está controlada por el argumento S de la cláusula matriz. Según Loos (1999), la lengua kapanawa carece de estos marcadores, siendo - as $y$-son empleados tanto para eventos previos como simultáneos (ver Cuadro 2). Debido a limitaciones de espacio, presentamos aquí oraciones ilustrativas correspondientes a dos lenguas, representativas de distintos subconjuntos Pano.

Amawaka (Sparing-Chávez 1998:460)
[hii rira-rïa-?i]-min
han pakïi-xo-?nì
árbol tumbar-tumbar-SIM.SI.S-TEMA él caer-3PASD-DECL
'[Mientras tumbaba los árboles], él se cayó'.

(23) [hiri-kin]-min

non

hato

ninkaa-ki-?ni. comer-SIM.SI.A-TEMA nosotros ellos escuchar-1PASD-DECL '[Mientras comíamos], nosotros los escuchamos'.

Kashibo-Kakataibo (Zariquiey 2011)
(24) a
Buan-i
$\mathrm{ka}$
kwan-(a)ki-s-a.
eso.O traer-SIM.SI.S NAR.3 ir-PASD.REM-3-no.PROX
'Trayendo eso, ellos se fueron'. (p. 575) 
En la oración que sigue, las dos cláusulas marcadas por -kin modifican la cláusula principal finita.

\author{
?a-pun-kin kaisa ?itsi ñantan ?uş-kin \\ hacer-mismo.día-SIM.SI.A NAR.REP.3 otra tarde dormir-SIM.SI.A \\ ?a-(a)ki-s-in. \\ hacer-PASD.REM-3-PROX \\ 'Dicen que haciéndolo temprano, durmiendo otra tarde, lo hizo'. (p. 578)
}

\title{
2.1.3. Eventos Posteriores
}

Como puede observarse en el Cuadro 2, las lenguas shipibo-konibo, matsés, kashibo-kakataibo y amawaka han desarrollado marcadores de $\mathrm{CP}$ específicos de sujetos idénticos correspondientes a eventos posteriores al evento en la cláusula matriz (en kapanawa un solo marcador es usado para indicar orientación hacia S y A); estructuralmente amawaka y parcialmente matsés emplean morfología que incluye los marcadores empleados para eventos simultáneos. Los marcadores de eventos posteriores orientados hacia A en shipibo-konibo y kashibo-kakataibo difieren de uno de los marcadores correspondientes en matsés en que los primeros muestran la secuencia adicional -so $\sim-s u$ en -noson $\sim-n u s u n$ mientras que matsés registra simplemente $-n u n^{18}$. Debido a limitaciones de espacio, presentamos aquí oraciones ilustrativas correspondientes a solo una de las lenguas seleccionadas para el estudio.

Kashibo-Kakataibo (Zariquiey 2011)

$\begin{array}{llll}\text { [tsi } & \text { mikamanus] } & \text { kaisa } & \text { t firikinin rara } \\ \text { tsi } & \text { mikama-nus] } & \text { kaisa } & \text { t firikin=n rara } \\ \text { fuego.ABS } & \text { robar-POST.SI.S } & \text { NAR.REP.3 loro.pequeño=GEN ancestro.ABS }\end{array}$

tsóakişa tsi kwißi utinßuas

tsot-aki-s-a tsi kwi $\beta$ i utin $\beta u$-as

vivir-PASD.REM-3-no.PROX fuego cerca estar.pensativo-PREV.SI.S

'Dice que, [para robar el fuego], el loro se sentó cerca, lamentablemente.' (p. 577)

(27)

$\begin{array}{llll}\text { [nae } & \text { Tanusun } & \text { kananuna mepain } & \text { Barin } \\ \text { nae } & \text { Ta-nusun } & \text { kananuna me=pain } & \text { Bari-i-n }\end{array}$

chacra:ABS hacer-POST.SI.A NAR.1PL tierra.ABS=primero buscar-IMPF-1/2

'[Para hacer chacra], primero tenemos que buscar un terreno'. (p. 579)

18 En kapanawa la forma -noşon indica orientación hacia el sujeto de la cláusula matriz, sin distinguir S de A. Evidentemente se trata de la forma orientada hacia A cuya función se extendió, desplazando a la forma orientada hacia S. 


\subsubsection{Concordancia del participante a nivel inter-oracional: conclusiones}

Las lenguas seleccionadas exhiben morfemas cognados de sujetos idénticos correspondientes a eventos conceptualizados como previos o simultáneos con respecto al evento de la cláusula matriz. Estos morfemas varían según la función sintáctica del argumento correferencial en la cláusula matriz; obviamente, este criterio se correlaciona con la transitividad de dicha cláusula. El kapanawa carece de marcadores especializados de eventos simultáneos ${ }^{19}$. Shipibokonibo, matsés, kashibo-kakataibo y amawaka han desarrollado marcadores específicos de sujetos idénticos correspondientes a eventos posteriores o subsecuentes; estructuralmente, amawaka y parcialmente matsés emplean en estos casos morfología que incluye los marcadores de eventos simultáneos. Los marcadores de eventos posteriores orientados hacia A en shipibo-konibo y kashibo-kakataibo por un lado, y matsés por otro, difieren en que los primeros muestran la secuencia adicional -so $\sim-s u$ en -noson $\sim$-nusun mientras que matsés exhibe simplemente -nun.

Otro aspecto importante a resaltar es la existencia de marcadores que indican correferencia con el objeto, como por ejemplo $\mathrm{O}=\mathrm{S} / \mathrm{A}$ en matsés, kashibo-kakataibo y shipibo-konibo, $\mathrm{S} / \mathrm{A}=\mathrm{O}$ en amawaka y $\mathrm{S} / \mathrm{A} / \mathrm{O}=\mathrm{O}$ en matsés y kashibo-kakataibo. Además, en esta última lengua encontramos marca de $\mathrm{CP}$ en los sufijos que indexan correferencialidad entre $\mathrm{O}$ y $\mathrm{S}\left(-k i_{\xi}\right)$, así como entre $\mathrm{O}$ y A (-kissun).

\subsection{Concordancia del participante a nivel intra-oracional}

En las cláusulas simples la CP puede atestiguarse en palabras o frases adjuntas que indican lugar y otras funciones comúnmente consideradas "adverbiales", tales como cuantificación distributiva, etapa de vida, benefactivo/ malefactivo, comitativo, manera, razón, etc. A diferencia de las expresiones de etapa de vida (por ejemplo, "de niño"), las expresiones de tiempo (por ejemplo, "ayer") predican sobre el evento en su totalidad y no sobre un participante en particular. Crucialmente, los adjuntos de tiempo no suelen ser sensibles a la $\mathrm{CP}^{20}$. Es necesario mencionar que son muy pocas las lenguas Pano en las que la $\mathrm{CP}$ intra-oracional ha sido descrita con cierto detalle. Es por este motivo, así como por los límites de espacio, que no ilustramos los diferentes tipos de adjuntos para cada subconjunto Pano. En esta sección presentaremos primero las expresiones locativas (2.2.1), para luego ofrecer un panorama de los demás

19 Los morfemas -kin e - $i$ están presentes en la lengua pero sus funciones han cambiado de manera significativa. Loos (1999) les asigna la glosa CONJ o conjunción, como se puede ver en (28)-(30).

20 Como veremos más adelante, las lenguas Pano norteñas presentan algunas aparentes excepciones. 
tipos de adjuntos que exhiben el fenómeno que nos ocupa (2.2.2). Además, si consideramos la orientación hacia el participante y la orientación hacia el evento como dos extremos de un continuum, observamos que los diferentes tipos de adjuntos se ubican en puntos distintos del mismo.

\subsubsection{Adjuntos locativos}

Cuando predican acerca del participante A, los adjuntos locativos llevan una marca explícita que coincide con el morfema de sujetos idénticos -son sun (PREV.SI.A); la orientación hacia $\mathrm{O}$ no es indicada morfológicamente. Por otro lado, el complemento del verbo 'venir' recibe el marcador - as que indica orientación hacia el participante $S$; este marcador coincide con un segundo morfema de sujetos idénticos (PREV.SI.S). En ciertos casos, -as puede coocurrir con otros verbos intransitivos (como se aprecia en y ; para ejemplos en shipibo-konibo, véanse Valenzuela 2003 y 2005a).

KAPANAWA: lugar (Loos 1999:239-240)

Como se mostró mediante las oraciones chákobo (1) y (2), cuando existe más de un participante central, la $\mathrm{CP}$ despeja la ambigüedad potencial en cuanto al argumento que ejerce control sobre el adjunto locativo. Veamos los siguientes ejemplos kapanawa:

(28) hano ?ano his-kin

ahí:O majás ver-CONJ

'Él vio el majás ahí' (el locativo predica acerca del participante O).

(29) hano-son his-kin

ahí-A ver-CONJ

'Él lo vio desde allí'. (el locativo predica acerca del participante A)

(30) hano?-as haw-i

ahí-S venir-CONJ

'Él vino de ahí'. (el locativo predica acerca del participante S)

MATIS: lugar (Fleck 2010:52)

(31) idantfa-n-sun inbi nawa-0 is-bo-k bote-LOC-A 1:ERG no.indígena-ABS ver-PAS.REC-INDIC:1/2 'Yo vi al no-indígena desde el bote'. (el locativo predica acerca de A)

(32) idantfa-n inbi nawa-0 is-bo-k.

bote-LOC:O 1:ERG no.indígena-ABS ver-PAS.REC-INDIC:1/2

'Yo vi al no-indígena en el bote'. (el locativo predica acerca de $\mathrm{O}$ ) 
MATSÉS: lugar (Fleck 2003:584-585)

(33)

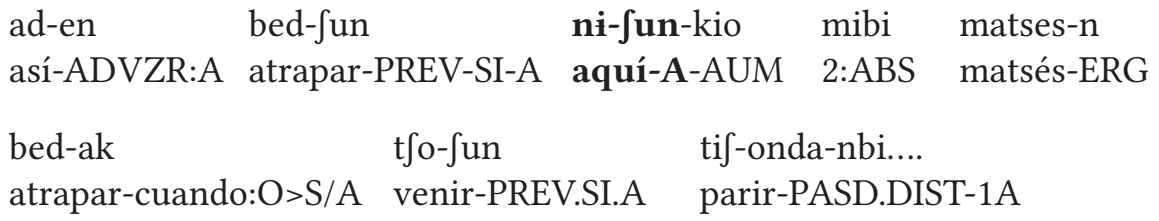

'Después de que los matsés me capturaron así, te parí aquí después de que me capturaron y yo vine'. (el locativo predica acerca de A)

(34) afik ni-wif-kio-bi mibi kani-e-k

luego aquí-S-ENF 2:ABS crecer-no.PASD-INDIC

'Luego naciste aquí'. (p. 585) (el locativo predica acerca de S)

KASHIBO-KAKATAIBO: lugar (Zariquiey 2011:472)

En la oración el morfema - $a$ es interpretado como indicador de CP con orientación hacia O. En kashinawa (Montag 2008 y oración ) y shipibo-konibo (Valenzuela 2003) el morfema equivalente es analizado como ablativo, en tanto que la orientación hacia $\mathrm{O}$ recibe marca cero.

uni $=\mathrm{n} \quad \mathrm{ka} \quad \boldsymbol{\beta a k a}=\mathbf{n u}=\mathbf{s u n} \quad \mathrm{t} \int \mathrm{s} \mathrm{su}$ ?a-a-s-a.

hombre=ERG NAR.3 río $=\mathbf{L O C}=\mathbf{A}$ venado.ABS matar-PERF-3-no.PROX 'El hombre, estando en el río, mató al venado'.

(36) uni=n ka $\quad$ a aka $=\mathbf{n u}=\mathbf{a}$ t $\int a s u \quad$ ?a-a-s-a.

hombre=ERG NAR.3 río=LOC $=\mathbf{O}$ venado.ABS matar-PERF-3-no.PROX

'El hombre mató al venado, el cual estaba en el río'.

(37) uni ka $\quad \boldsymbol{\beta a k a}=\mathbf{n u}=\mathbf{a s} \quad \mathrm{kwa}-\mathrm{i} \quad$ i-a-s-a.

hombre.ABS NAR.3 río=LOC=S jugar-SIM.SI.S ser-PERF.3-no.PROX

'El hombre, estando en el río, estaba jugando'.

KASHINAWA: adentro de (Montag 2008:44-46)

(38) in hiwi-tan-sun in kini-mis-ki.

1:NOM casa-dentro.de-A $1: \mathrm{NOM}$ escribir-HAB-COMPL

'Escribo dentro de mi casa'. (Montag 2008:45)

A diferencia de lo que encontramos en otras lenguas Pano, en kashinawa el complemento locativo del verbo 'venir' no lleva el marcador - as sino simplemente - a (interpretado en esta lengua como ablativo, ver también Valenzuela 2003 para un análisis análogo en shipibo-konibo). Esto es consistente con la pérdida de la consonante retrofleja sibilante en posición 
de coda que registramos para el morfema de sujetos idénticos - $a$ (remítase a la oración ). Este proceso de elisión fonológica tiene como consecuencia la pérdida de la distinción formal entre los locativos orientados hacia $\mathrm{S}$ (ejemplo ) y aquellos orientados hacia $\mathrm{O}$ (ejemplo ):

$\begin{array}{llll}\text { (39) } & \text { ni } & \text { midanua } & \text { hu-ikiki. } \\ \text { ni } & \text { miran-anu-a } & \text { hu-ikiki } \\ \text { monte } & \text { adentro-LOC-ABL } & \text { venir-INCOMPL.3.DECL } \\ \text { 'Él viene del monte'. (Montag } & \text { 2008:35) }\end{array}$

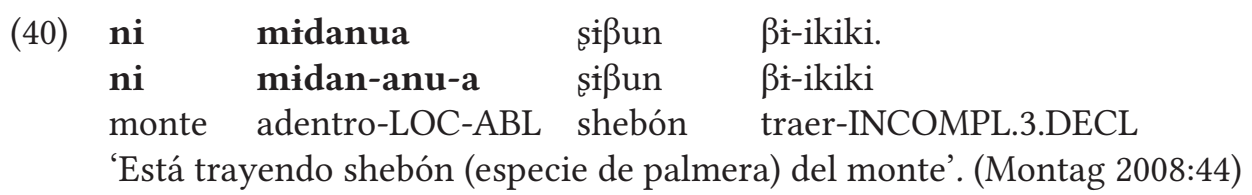

Mientras que en kashinawa, yaminawa y shipibo-konibo el complemento locativo del verbo 'traer' es codificado como ablativo y está orientado hacia $\mathrm{O}$ (es decir, recibe el marcador - $a$, como en ), en matsés, kashibo-kakataibo y chákobo, este es codificado como locativo y muestra orientación hacia A (es decir, recibe el marcador -nu-sun o sus equivalentes como en ). Veamos las siguientes oraciones de la lengua matsés.

MATSÉS (Kneeland 1979)

$$
\begin{array}{lll}
\text { aton } & \text { tied-no-i } & \text { t } 0 \text { o-e-k. } \\
\text { POS3 } & \text { chacra-LOC-S } & \text { venir-no.PASD-INDIC }
\end{array}
$$

'Él viene de su chacra'. (Kneeland 1979:1)

(42) tied-no-fon pachid $\beta \dot{\mathrm{i}}-\mathrm{o}-\int$.

chacra-LOC-A yuca:ABS traer-PASD-3

'Él/ella trajo yuca de la chacra'. (Kneelad 1979:35)

\subsubsection{Otros tipos de adjuntos}

En esta sección se demuestra que la $\mathrm{CP}$ no se halla restringida a las expresiones de lugar, sino que opera en una gama amplia de adjuntos. En estas construcciones es posible distinguir dos patrones principales: los adjuntos marcados mediante -ass/-son (o sus equivalentes) y aquellos marcadores por -i/-kin (o sus equivalentes). Como se trató en las secciones 2.1.1 y 2.1.2, estos morfemas funcionan como marcadores de sujetos idénticos, adhiriéndose a cláusulas que codifican, respectivamente, eventos previos y eventos simultáneos con respecto al evento en la cláusula matriz. 
CHÁKOBO: razón (Zingg 1998:42)

(43) hini-?as tsi mi ho-?a?

por.qué-S PRTCL 2.NOM venir-COMPL

¿Por qué viniste?’

(44) hini-so sobo a-jama-?ai?

por.qué-A casa hacer.TR-NEG-INCOMPL:INT

¿¿Por qué no construyes una casa?'

CHÁKOBO: cuantificación distributiva (Valenzuela 2005b:190)

(45) rabi-?as ha paßi-ká-kí.

dos-S 3:NOM bailar-SUJ.PL-COMPL

'Ellos bailaron de a dos/Ellos dos bailaron'.

(46) rabi-so sobo ha a-ká-ki.

dos-A casa 3:NOM AUX.TR-SUJ.PL-COMPL

'Ellos construyeron la casa entre dos/Ellos dos construyeron la casa'.

MATSÉS: cuantificación distributiva (Fleck 2003:593, hablantes mayores)

(47) abitedi-fun matses-n pe-aid

todos-A matsés-ERG comer-NMLZR.PAC

pofto ne-e-k.

mono.choro ser-no.PASD-INDIC

'Los monos choros son comidos por todos los matsés'.

(48) abitedi-wif nid-o- $\int$.

todos-S ir-PASD-3

'Todos fueron'.

KASHIBO-KAKATAIBO: distribución no cuantitativa

(49) sanu-sun ka ?a-a-s-a

mujer-A NAR.3 hacer-PERF-3-no.PROX

'Entre mujeres lo hicieron'. (Zariquiey 2011:478)

CHÁKOBO: compañía (Valenzuela 2005b:196-197)

(50) no $\quad \boldsymbol{\beta i t a - s o}$ siki tima-ki.

1PL COM-A maíz moler-INCOMPL

'Él muele maíz con nosotros'. 
(51) i-bita-2as ina orikí ${ }^{21}-k \dot{k}$.

1-COM-S perro comer.INTR-COMPL

'El perro comió conmigo'.

YAMINAWA: propietivo 'con' (Faust y Loos 2002:42)

(52) $\tilde{1}$

1.NOM hacha-PROP ir-PASD.ayer

'Fui con mi hacha (ayer)'.

(53) foskiti-ja-şo pi-i.

escopeta-PROP-A comer-PROGR

'Él está comiendo con su escopeta (en su posesión) (indica que tiene su escopeta a su lado o a cuestas)'.

Una construcción que involucra el propietivo -ja también puede tener una interpretación instrumental, como lo ilustra el siguiente par de oraciones chákobo,

CHÁKOBO: instrumento (Valenzuela 2005b)

(54) mikí-ja-şo waí nili ha a-ki.

mano-PROP-A chaco:LOC monte:ABS 3:NOM AUX.TR-COMPL

'Él cultivó el chaco con sus manos'.

(55) raßí Jinó a-ki pia-ja-so-ma.

Rabi:ERG mono AUX.TR-COMPL flecha-PROP-A-NEG

'Rabi mató al mono sin flecha'.

o incluso una interpretación de condición emocional, como se ve en la siguiente oración shipibo-konibo:

SHIPIBO-KONIBO: condición emocional (Valenzuela 2005a:282)

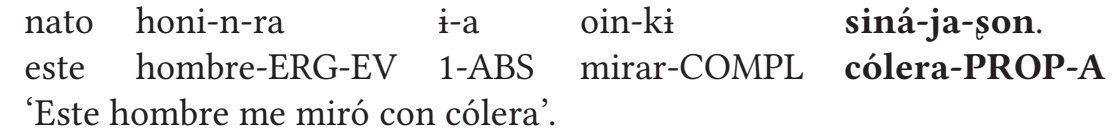

SHIPIBO-KONIBO: complemento de interés (Valenzuela 2005a:278-279)
(57) ha-ra i-on-mi-a-s- $\beta$ i wano-ki.
3:ABS-EV 1-INTERÉS-LOC-ABL-S-ENF casarse.un.hombre-COMPL
'Él se casó (con otra mujer) a pesar de estar conmigo'.

21 Se trata de un verbo intransitivo, en tanto que $p i$ - 'comer' es transitivo. 
En shipibo-konibo el verbo kinan- 'vomitar' se comporta como un verbo transitivo en el sentido de que su sujeto es marcado con el caso ergativo e impone las concordancias correspondientes a $\mathrm{A}^{22}$ :

(58) mi-on-son

$$
\text { Baki-n kinan-a. }
$$

2-INTERÉS-A niño-ERG vomitar-PARTCP

'El niño vomitó estando bajo tu cuidado'.

SHIPIBO-KONIBO: etapa de vida, condición (Valenzuela 2005a:279-280)

(59) ha-rißa-ki

ßaki tfia-a-ti,

existir-REPE-COMPL niño flaquito-hacer-INF

$\beta a k i-s-\beta i$

too-ki-tian

poko-mi-a- $\beta \mathrm{i}$

ßaki masko a-ti.

embarazarse-PREV-SD vientre-LOC-ABL-ENF niño pequeño hacer-INF

'También hay (una planta con poderes especiales) para que el feto sea muy pequeño cuando una niña sale embarazada a temprana edad'.
(60) mapó a-kin
pio-ti
r-iki

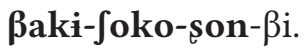
barro hacer-SIM.SI.A empezar-INF EV-COP niña-DIM-A-ENF
'Para hacer cerámica hay que empezar desde niña'.

La siguiente oración shipibo-konibo es interesante, ya que el empleo del temporal -tian en vez de un marcador de CP indica que la frase adjunta no predica acerca de uno de los participantes de manera específica:
(61) i-n-ra
onan-ki
sankin $\beta$ ari $\beta$ aki-tian- $\beta$ i.
1-ERG-EV conocer-COMPL Sanken Bari niño-TEMP-ENF
'Yo conocía a Sanken Bari cuando yo era niño / cuando Sanken Bari era niño’.

CHÁKOBO: referencia, 'acerca de' (Valenzuela 2005b:190)

En la oración chákobo que sigue, el predicado joa-kas- es intransitivo como lo indica el marcador de concordancia del participante en el adjunto 'acerca de nuestra comunidad':

(62) hariapari tsi noba comunidad ta?ina-Pas

primero VNC nuestra comunidad acerca.de-S

joa-kas-kia.

contar-DES-INCOMPL.1

'Primero quiero contar(les) acerca de nuestra comunidad'.

22 Para una discusión acerca de la transitividad del verbo kinan-, ver Valenzuela (2003:577579). 
Al comparar las expresiones que llevan los marcadores - ass/-sun (o sus equivalentes) observamos que estas son propiamente orientadas hacia un participante, en tanto que aquellas que reciben -i/-kin (o sus equivalentes) muestran esta característica en menor grado, orientándose (más) hacia el evento. Veamos las formas shipibo-konibo kimifa-son y kimifa-a-kin en las oraciones 3) y 4); mientras la primera predica del participante en función de A, la segunda predica acerca del evento. Nótese también que los adjuntos que toman - $k i n$ pueden requerir de la presencia del auxiliar transitivo $a(k)$-.

SHIPIBO-KONIBO (Valenzuela 2003)

$\begin{array}{llll}\text { ha- } \beta \text { aoon-ra } & \text { kimifa-son- } \beta \text { o } & \text { naranfa } & \beta \dot{i}-k \dot{ } \\ \text { 3-PL:ERG-EV } & \text { tres-A-PL } & \text { naranja:ABS } & \text { conseguir-COMPL }\end{array}$

'Ellos agarraron naranjas (actuando) en grupos de tres'.

$\begin{array}{llll}\text { ha-n-ra } & \text { kimifa-a-kin } & \text { pi-a } & \text { iki. } \\ \text { 3-ERG-EV } & \text { tres-hacer-SIM.SI.A } & \text { comer-PRTCP } & \text { AUX }\end{array}$ 'Él comió tres veces'.

Veamos otros tipos de adjuntos que involucran los morfemas -i/-kin (o sus equivalentes) y exhiben un menor grado de orientación hacia un participante que aquellos marcados por $-a s /$-son.

KASHIBO-KAKATAIBO: manera (Zariquiey 2011:354)

(65) kwan-sun kana upit-o-kin $\dot{\mathrm{i}}=\mathrm{n}$ ?atsa pi-i-n ir-PREV.SI.A NAR.1SG bonito-FACT-A 1 SG=A yuca.ABS comer-IMPF-1/2 'Habiendo ido, yo estoy comiendo yuca muy bonito'.

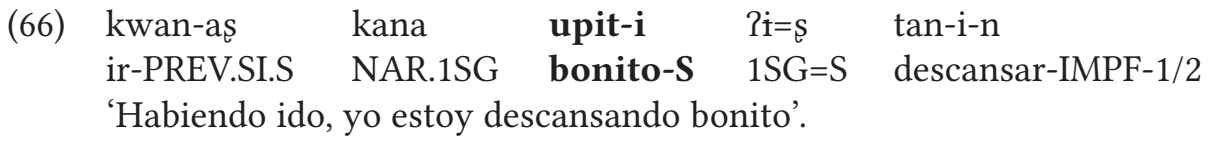

MATSÉS: manera (Fleck 2010:50-51)

(67) debi-0 kumanpen-ek kapu-o- $\mathrm{s}^{23}$.

Davy-ABS intensamente-S caminar-PASD.REC-3

'Davy caminó rápido/fuerte'.

En matsés, -en alterna con -kin a nivel inter-oracional (Fleck 2003:1080, 1083; ver también el Cuadro 2 arriba). En la CP intra-oracional se emplea la primera forma:

23 En esta publicación se consigna la consonante retrofleja sibilante en la lengua matsés, a diferencia de Fleck (2003). 
(68) debi-n kumanpen-en kues-o-s-i.

Davy-ERG intensamente-A golpear-PASD.REC-3-1O

'Davy me golpeó fuerte'.

Como se señaló en 2.2. en general las lenguas Pano no exhiben CP en las expresiones temporales. Sin embargo, en las lenguas Pano norteñas matsés y matis se ha reportado el uso de morfología de CP con ciertos adverbios de tiempo. Es posible que estos adverbios hayan surgido originalmente de verbos del tipo 'amanecerse'24.

MATSÉS: tiempo Kneelad (1979:205)

(69)

$\begin{array}{ll}\text { abadiad-af } & \text { t } \int o-e b i . \\ \text { pasado.mañana-S } & \text { venir-1SG.ABS }\end{array}$

'Voy a venir pasado mañana ${ }^{25}$ '.

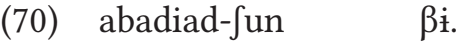

pasado.mañana-A traer

'Tráelo pasado mañana'.

El Cuadro 3, adaptado de Valenzuela (2005a), resume el ámbito semántico de los adjuntos que reciben $\mathrm{CP}$ en Pano, así como los marcadores involucrados recurrentes en las lenguas seleccionadas. Nótese que en este trabajo no se ha tratado la concordancia del participante en las conjunciones. Estas últimas suelen derivarse de expresiones locativas.

Cuadro 3. Adjuntos con concordancia del participante en Pano y morfemas recurrentes

\begin{tabular}{|c|c|c|c|}
\hline $\begin{array}{c}\text { Concordancia } \\
\text { con S }\end{array}$ & $\begin{array}{c}\text { Concordancia } \\
\text { con A }\end{array}$ & $\begin{array}{l}\text { Adjuntos internos } \\
\text { a la cláusula }\end{array}$ & $\begin{array}{c}\text { Cláusulas } \\
\text { marcadas de } \\
\text { sujetos idénticos }\end{array}$ \\
\hline $\begin{array}{l}-(?) a s \\
-? a x \\
-a \int \\
-a(\text { kashinawa }) \\
- \text { wi } \\
-s\end{array}$ & $\begin{array}{l}\text {-son } \\
\text {-sõ } \\
\text {-sun } \\
\text {-sun } \\
\text {-xon } \\
\text {-so }(\mathrm{CH})\end{array}$ & $\begin{array}{l}\text { locativo/alativo (sólo A) } \\
\text { "ablativo" (sólo S) } \\
\text { manera/razón } \\
\text { cantidad/distribución } \\
\text { propietivo/instrumental } \\
\text { privativo (sólo A) }\end{array}$ & Evento previo \\
\hline
\end{tabular}

24 Compárese por ejemplo el término abadiad traducido como 'pasado mañana' en Kneeland (1979:205) con el verbo intransitivo badiad- 'amanecer (el día), despertarse en la madrugada, trasnochar' en Fleck et alia (2012:39).

25 Este adverbio es traducido como 'el siguiente día' en Fleck et alia (2012:25). 


\begin{tabular}{|c|c|c|c|}
\hline & & $\begin{array}{l}\text { benefactivo/malefactivo } \\
\text { etapa de vida } \\
\text { pronombre enfático } \\
\text { comitativo } \\
\text { referencia } \\
\text { conjunción }\end{array}$ & \\
\hline $\begin{array}{l}-? i \\
-i \\
-e k\end{array}$ & $\begin{array}{l}-k i n \\
-k i \\
-k i(\mathrm{CH}) \\
-e n\end{array}$ & $\begin{array}{l}\text { Manera } \\
\text { cantidad/distribución } \\
\text { conjunción }\end{array}$ & $\begin{array}{l}\text { Evento } \\
\text { simultáneo }\end{array}$ \\
\hline $\begin{array}{l}-n o s \\
-n u s \\
-n u \int \\
-n u e k \\
-k a t z i \\
-x a n h n i\end{array}$ & $\begin{array}{l}\text {-noson } \\
\text {-nusun } \\
\text {-nun } \\
\text {-nuen } \\
\text {-xanhkin }\end{array}$ & -- & Evento posterior \\
\hline
\end{tabular}

\subsubsection{Concordancia del Participante a nivel intra-oracional: Conclusiones}

En esta sección se ha demostrado que la $\mathrm{CP}$ opera al interior de la cláusula en lenguas pertenecientes a las diferentes subdivisiones de la familia Pano. Los adjuntos involucrados expresan lugar así como una variedad de funciones adicionales, tales como cuantificación distributiva, distribución, etapa de vida, condición emocional, compañía, instrumento, benefactivo/ malefactivo, razón, manera y referencia. Los adjuntos temporales no exhiben $\mathrm{CP}$, lo cual es compatible con el hecho de que estos predican acerca del evento en su totalidad y no acerca de un participante específico. Algunas expresiones temporales en las lenguas norteñas constituyen aparentes excepciones a esta generalización; por ejemplo, el adverbio matsés abadiad 'pasado mañana/el día siguiente'. Sin embargo, es probable que estas expresiones tengan un origen verbal. Los morfemas que codifican $\mathrm{CP}$ a nivel intra-oracional son formalmente (casi) idénticos a aquellos que expresan $\mathrm{CP}$ a nivel inter-oracional, específicamente los morfemas de sujetos idénticos correspondientes a eventos previos y simultáneos con respecto al evento en la cláusula matriz. Asimismo, se propuso que la orientación hacia el participante y la orientación hacia el evento pueden ser vistos como los extremos de un continuum, y que los adjuntos marcados por -as/-son (o sus equivalentes) exhiben un mayor grado de orientación hacia el participante que los marcados por -i/-kin (o sus equivalentes). En conclusión, podemos ver que la $\mathrm{CP}$ constituye un fenómeno unitario, que se manifiesta en dos planos sintácticos distintos, que aquí hemos llamado inter- 
oracional e intra-oracional. El primero forma parte de los sistemas de cambio de referencia (switch-reference). Por lo tanto, las descripciones de las lenguas Pano deben dar cuenta de este importante rasgo en su totalidad y no limitarse al tratamiento del nivel inter-oracional.

\section{La concordancia del participante desde una perspectiva interlingüística}

Semántica y formalmente, los adjuntos con $\mathrm{CP}$ en Pano son comparables a los predicados secundarios descriptivos (depictive secondary predicates) en otras lenguas, tales como "molestas" y "crudo" en las oraciones españolas "Alejandrina y Patricia se fueron molestas" y "Alejandrina y Patricia comieron el pescado crudo", que concuerdan en género y número con el participante sobre el cual predican. Al igual que los predicados secundarios del español, inglés y otras lenguas, los adjuntos Pano predican sobre un participante, son externos al sintagma nominal, son opcionales y expresan una situación que es válida en el momento en que la eventualidad expresada por el verbo tiene lugar (es decir, ambas situaciones son vistas como contemporáneas, lo cual diferencia a los predicados secundarios de los resultativos) (Schultze-Berndt y Himmelmann 2004:77-79). La CP, pues, es comparable a la concordancia de caso y/o género-número en lenguas como el griego clásico y el latín, y hasta cierto punto el español.

Sin embargo, como se señala en Valenzuela (1999, 2003, 2005a), la CP se asemeja más a las instancias de concordancia de caso en adjuntos reportadas para varias lenguas originarias de Australia que, como las Pano, tienen sistemas de marcación de caso ergativo-escindidos. Así, la orientación hacia un participante en función de $\mathrm{S}$ tiene una expresión formal distinta de la orientación hacia un participante en función de A. El sistema de CP Pano exhibe, no obstante, peculiaridades interesantes que lo desvían de los casos prototípicos de predicados secundarios. En primer lugar, como se ilustró en 2.2, en las lenguas Pano la $\mathrm{CP}$ es atestiguada en una amplia gama de adjuntos, incluyendo áreas semánticas en las que la presencia de este rasgo resulta inesperada. Por el contrario, no hallamos $\mathrm{CP}$ con adjetivos simples o frases adjetivales, que representan las instancias más ampliamente reconocidas de predicados secundarios (Himmelmann y Schultze-Berndt, 2005). Esto es así a pesar de que las lenguas Pano poseen una clase adjetival y los adjetivos pueden funcionar como predicados principales.

Una segunda peculiaridad del sistema Pano es que, a diferencia de casi todas las lenguas que exhiben concordancia en los adjuntos, carece de concordancia alguna al interior del sintagma nominal. Por lo tanto, la CP no puede ser explicada como una extensión de la concordancia al interior del sintagma nominal (Valenzuela 2003:879). Esto es importante, ya que las lenguas Pano 
representan un claro contra-ejemplo a un casi universal implicacional que podría decir lo siguiente: "Si en una lengua los adjuntos exhiben concordancia con un argumento de la cláusula, entonces también los modificadores al interior del sintagma nominal concuerdan obligatoriamente con el núcleo" (Schultze-Berndt y Himmelmann 2004:83).

En tercer lugar, la morfología que indica $\mathrm{CP}$ en Pano es opaca sincrónicamente (especialmente en chákobo). Estudios comparativos han demostrado que marcadores como -son y -?as habrían surgido diacrónicamente de la combinación de un marcador de caso oblicuo o secuencial y un marcador de caso adicional que concordaba con las funciones $\mathrm{S}, \mathrm{A}$ u O del argumento controlador (Valenzuela 2003, capítulo 20; véase también la sección 4 abajo). En las lenguas actuales, sin embargo, los marcadores de CP no corresponden a ningún otro morfema flexivo (con excepción parcial del amawaka (SparingChávez 1998)).

En cuarto lugar, a diferencia de los predicados secundarios prototípicos que son construcciones internas a la cláusula, la $\mathrm{CP}$ en Pano también ocurre a nivel de la combinación de cláusulas. Como se ha señalado en 2.2.3, los mismos morfemas que indican orientación semántica de los adjuntos hacia A ó S ocurren al final de ciertas cláusulas dependientes como marcadores de sujetos idénticos; por lo tanto, la morfología de CP forma también parte del sistema de cambio de referencia de la lengua.

\section{Desarrollo diacrónico del sistema de concordancia del participante en Pano}

A partir de la inspección comparativa presentada en 2.1 y 2.2 es posible arribar a dos importantes conclusiones. En primer lugar, la $\mathrm{CP}$ tanto en el nivel inter-oracional como en el nivel intra-oracional está presente en todas las lenguas examinadas, representantes de diferentes subconjuntos de la familia Pano. Además los marcadores de CP son formalmente idénticos o muy similares en todas las lenguas. Por lo tanto, podemos postular este rasgo como parte de la gramática del Proto-Pano ${ }^{26}$.

En segundo lugar, observamos que la distribución global de los marcadores de $\mathrm{CP}$ presenta una configuración tripartita: los marcadores que codifican orientación hacia A terminan en nasalización o /n/ (con excepción del chákobo, según la regla de pérdida de este rasgo o segmento); los marcadores orientados hacia $\mathrm{S}$ terminan en /s/ ó /i/, o sus formas cognadas (con excepción de la pérdida de la sibilante retrofleja en algunos adjuntos kashinawa); y la orientación hacia $\mathrm{O}$ es indicada mediante cero (la forma - $a$ en la oración (36) del

26 No obstante los aparentes procesos de simplificación del sistema por los que podría haber atravesado la lengua kaxararí. 
kashibo-kakataibo correspondería a un marcador oblícuo y no a un morfema de $\mathrm{CP}$ ). Hasta cierto punto esto es reminiscente a los sistemas de marcación de caso con distribución ergativa que hallamos en las lenguas Pano, al menos a nivel de los nombres, en los cuales el caso ergativo es indicado mediante $/ \mathrm{n} / \mathrm{o}$ nasalización, en tanto que el caso absolutivo no es marcado morfológicamente. Sin embargo, es más iluminador comparar la CP con los sistemas de marcación de caso tripartitos que encontramos en ciertas lenguas Pano,específicamente kashibo-kakataibo y amawaka, en los que el sujeto intransitivo se indica mediante $-s$ (kashibo-kakataibo) o - $x$ (amawaka), mientras que el sujeto transitivo y el objeto se marcan mediante $-n$ y -0 respectivamente. Es decir, podemos concluir que el sistema de CP Pano está diacrónicamente relacionado a un sistema de concordancia de caso. Una consecuencia importante de este análisis para la reconstrucción del Proto-Pano es la probable existencia de un sistema de caso tripartito en ciertas áreas de la gramática de la proto-lengua.

\subsection{Reconstrucción de los marcadores de concordancia del participante}

Valenzuela (2003: capítulo 20) propone una reconstrucción de los marcadores de CP compartidos por las lenguas Pano: -as, -son, -kin, -nos, -non, -noson o sus formas $\operatorname{cognadas}^{27}$. Estos morfemas no son (completamente) analizables en las lenguas actuales; probando ser especialmente opacas en chákobo, pero más transparentes en amawaka y kashibo-kakataibo. El análisis comparativo revela que los morfemas de $\mathrm{CP}$ son el resultado de la combinación y fusión de dos capas de marcadores:

\begin{tabular}{|c|c|c|}
\hline- SO $\sim-$ Su $\sim-\mathrm{Xo}$ & $+-n$ & controlado por $\mathrm{A}$ \\
\hline$-\mathrm{ki}$ & $+-n$ & controlado por $\mathrm{A}$ \\
\hline- no $\sim-$ nu & $+-n$ & controlado por $\mathrm{A}$ \\
\hline$-1 \mathrm{a} \sim-\mathrm{a}$ & $+-s$ & controlado por $\mathrm{S}$ \\
\hline- no $\sim-$ nu & $+-s$ & controlado por S \\
\hline$-1 \mathrm{a} \sim-\mathrm{a}$ & +-0 & controlado por $\mathrm{O}$ \\
\hline so $\sim$ su -xo & +-0 & controlado por \\
\hline
\end{tabular}

\subsubsection{Primera capa: marcadores de caso periféricos}

Siguiendo el principio de que el cambio se dio desde los significados más concretos a los más abstractos, y desde los contextos nominales a cláusulas nominalizadas, se propone lo siguiente para el Proto-Pano:

${ }^{*}-s o \sim{ }^{*}$ su secuencial $>$ pasado, completivo, perfecto

27 No se discute el posible origen del marcador de sujeto idéntico -(Pi) -ek. 
En las lenguas examinadas la secuencia /so/ o su forma cognada es parte de un morfema de sujeto idéntico que incluye un significado secuencial ('después de'). Además, -son y sus variantes son necesariamente controlados por el argumento $\mathrm{A}$, en tanto que -so por sí solo es controlado por $\mathrm{O}$ en amawaka (PREV.S/A=O) y matsés (PREV.S/A/O=O).

Las formas $-s o,-x o$, $-s u$ son marcadores de tiempo-aspecto en lenguas pertenecientes al grupo "Purús", según Valenzuela (2003): -s $u$ ha sido analizado como pasado del mismo día en kashinawa (Montag 2008:26) y - $x o$ como pasado perfectivo narrativo correspondiente a la tercera persona en amawaka (Sparing-Chávez 1998:447).

En la lengua sureña chákobo, so es una partícula "ecuacional" (Prost 1965) que participa de las construcciones copulativas. Es probable que * de ser un marcador secuencial a un marcador de tiempo pasado o aspecto completivo en kashinawa y amawaka.

\section{*-2a (¿ablativo?), secuencial < completivo, resultativo, pasado}

- $2 a$ (o su cognado) es analizado como ablativo en shipibo-konibo (Valenzuela 2003) y kashinawa (Montag 2008); sin embargo, no es claro que las otras lenguas Pano posean un marcador ablativo especializado. En las lenguas norteñas el marcador correspondiente cambia a /ít o /wi/ a nivel inter-oracional e intraoracional (Fleck 2010). Al parecer, en todas las lenguas Pano este marcador sigue al locativo en estructuras que codifican cierto tipo de procedencia. - $a$ ha sido descrito como morfema de aspecto completo en yaminawa (Faust y Loos 2002:119), acción terminada o completa en kashinawa (Montag 2008:36), perfecto de resultado en amawaka, pasado inmediato de primera persona en el aspecto de actualidad en amawaka ("immediate past first person actuality aspect", Sparing-Chávez 1998:447), participio completivo en shipibo-konibo (Valenzuela 2003) y, seguido por /k/ (es decir, -ak), nominalizador de acción o pasado reciente inferencial en matsés (Fleck 2003). ${ }^{*}$-?as habría formado parte del Proto-Pano, como un marcador secuencial o de aspecto completo.

\section{*-ki locativo no específico $>$ simultaneidad, incompletivo}

Shell propone la existencia en Pano-Reconstruido del locativo no específico o aproximativo * $k i$ (1975:143). Actualmente, las lenguas shipibo-konibo, kapanawa, kashinawa, yaminawa, amawaka y chákobo poseen un morfema locativo que refleja esta forma y función. Sin embargo, Shell no discute la relación de este proto-morfema del Pano-Reconstruido con los locativos - $m i$ del kashibo-kakataibo y matsés. Aunque estas formas parecen estar diacrónicamente relacionadas a ${ }^{*}-k i$ no nos es posible explicar el cambio de consonante. 
Además de su función de locativo no específico o aproximativo, Valenzuela (2003) agrega que -ki también se adhiere a los objetos indirectos en chákobo y kashinawa, y al estímulo de un predicado de emoción en shipibo-konibo, chákobo y kashinawa. Además, en yaminawa - $k i$ marca el estímulo de un predicado de emoción y malefactivo (Faust y Loos 2002:45) ${ }^{28}$.

Un morfema $-k i$ también indica aspecto incompletivo en chákobo (Valenzuela 2003:915) y aspecto de actualidad con tercera persona en amawaka (Sparing-Chávez 1998:446). Según Sparing-Chávez (1998:448), -Pki marca pasado inmediato perfectivo con la segunda y tercera personas en amawaka.

\section{${ }^{*}{ }_{-n o} \sim{ }^{*}-$ nu locativo/direccional $>$ posterior $>$ propósito, prospectivo}

La forma - no (-nu en matsés y - anu en kashinawa) significa 'en, a' en todas las lenguas examinadas. El morfema - no se reduce a - $n$ en shipibo-konibo (realizándose fonéticamente como nasalización en la vocal precedente) y también en otras lenguas de la familia. Como se mencionó arriba, la expresión de algún tipo de procedencia se basa en este locativo (al cual se añade - $a$ o su equivalente; véase el ablativo -nu- $a$ en kashinawa en y ). Además, $-n o \sim-n u$ funciona como indicador de propósito o aspecto prospectivo en las diferentes lenguas, formando parte de los marcadores de eventos posteriores en shipibo-konibo, kashibo-kakataibo y matsés. Finalmente, este locativo también funciona como indicador de sujetos diferentes (ver Cuadro 2).

En cuanto al marcador -(2)i ek empleado en la combinación de eventos (parcialmente) simultáneos solamente cabe mencionar que este podría guardar relación con un marcador de aspecto incompletivo.

\subsubsection{Segunda capa: marcadores de caso argumentales}

Una segunda capa de marcación de caso correspondiente a los argumentos del verbo habría sido agregada a las formas verbales nominalizadas que ya habían recibido un marcador de caso periférico o secuencial. Se trata de * $n$, ${ }^{*}-s \mathrm{y}{ }^{*}-0$ (Valenzuela 2003).

\section{* $-n$ marcador de sujeto transitivo A}

El morfema - $n$ (o nasalización) indica el caso ergativo en las diferentes lenguas Pano, especialmente en los nombres ${ }^{29}$. También marca al sujeto

28 En kashinawa, el morfema - $k i$ marca aspecto completo y el equivalente de los verbos 'ser' y 'estar' (Montag 2008:6); es posible que en este último caso nos encontremos frente a un morfema de origen distinto.

29 La excepción la constituye la lengua kaxararí, donde el ergativo es marcado mediante - $l$ tanto en nombres como pronombres (Valenzuela y Olivieira, notas de campo, LALI-UnB 2012). 
transitivo en los sistemas tripartitos del amawaka, kashibo-kakataibo y posiblemente iskonawa. Además, $-n$ marca el caso nominativo para la primera y segunda persona en kashinawa, yaminawa y otras lenguas del Purús, y forma parte de los pronombres nominativos en wariapano (Valenzuela 2000). Esta propuesta coincide con la de Loos (2005), y se diferencia de Shell (1975:103) quien postula *-mã como morfema de "referencia transitiva".

\section{*-0 marcador de objeto $\mathrm{O}$}

En todas las lenguas los objetos-pacientes llevan el marcador cero tanto a nivel de nombres como de pronombres (la única posible excepción son ciertos pronombres que llevan el sufijo - $a$ en shipibo-konibo, el cual ha sido analizado como morfema de caso absolutivo (Valenzuela 2003); sin embargo, según un análisis alternativo - $a$ formaría parte del pronombre).

\section{${ }^{*}-s$ marcador de sujeto intransitivo $S$}

Aunque como marcador de caso argumental - s está ausente en la mayoría de lenguas Pano, lo encontramos como indicador de sujeto intransitivo en los sistemas tripartitos de kashibo-kakataibo, amawaka (donde se realiza como - $x$ ) y posiblemente iskonawa. Shell (1975:115) no postula esta forma por sí misma para el Pano-Reconstruido, sino como parte del morfema de concordancia ${ }^{*}$-2asi. Shell reconstruye esta forma puesto que en marinawa, un idioma del grupo Purús, encontramos el marcador de sujetos idénticos - aşi en vez de -as. Sin embargo, -assi solamente se encuentra presente en marinawa y la variedad cercana sharanawa (considerados dialectos de la misma lengua).

Un sistema de marcación de caso tripartito con los marcadores propuestos arriba (o sus reflejos) existe en por lo menos dos lenguas Pano, amawaka y kashibo-kakataibo. Valenzuela (2003) llama la atención hacia el hecho de que estos sistemas exhiben un marcador expreso para el argumento $S$, un rasgo inusual en los sistemas tripartitos a nivel interlingüístico. Según SparingChávez (1998:446), el amawaka exhibe una configuración tripartita con sujetos pragmáticamente marcados y aspectos marcados (i.e., "aspectos que codifican eventos en los que alguien está o estará efectivamente involucrado" (mi traducción)), en los demás casos la lengua presenta una distribución ergativo-absolutiva o una neutra ${ }^{30}$. Los marcadores de caso correspondientes son - $x$ para S, $-n$ para A y -0 para O. Aunque el tema de la distribución del sistema tripartito amawaka no es tratado en detalle en la fuente consultada, observamos que los ejemplos proporcionados involucran solo nombres, y no pronombres.

$30 \mathrm{El}$ texto original dice: "In pragmatically neutral independent clauses, an ergativeabsolutive system is employed with unmarked aspect, but a neutral unmarked system with marked aspect" (Sparing-Chávez 1998:445-446). 
Como en amawaka, en la lengua Pano occidental kashibo-kakataibo también encontramos un sistema de marcación de caso tripartito con marcación explícita para S y A, y marcación cero para O. Sin embargo, es preciso establecer algunas diferencias entre estas dos lenguas. En primer lugar, en kashibo-kakataibo el sistema tripartito parece estar mucho más extendido que en amawaka. En efecto, en kashibo-kakataibo este sistema opera de manera irrestricta a nivel de los pronombres no enfáticos; los nombres siguen esta distribución cuando introducen un tópico anafórico (Zariquiey 2011). De lo dicho anteriormente podemos concluir que el sistema tripartito amawaka estaría asociado mayormente con los nombres, en tanto que el kashibo-kakataibo opera especialmente con los pronombres. En tercer lugar, el kashibo-kakataibo sería la única lengua Pano donde el contenido fonológico de ${ }^{*} s$ se habría preservado; los marcadores correspondientes a A y O son $-n$ y -0 respectivamente. Por último, un sistema de marcación de caso tripartito podría estar presente en la lengua iskonawa, con una manifestación formal similar a la del amawaka (Loos 1978:159). Lamentablemente, la información disponible sobre esta lengua es muy escasa. Como se muestra en el Cuadro 1, las lenguas amawaka y kashibo-kakataibo pertenecen a dos subdivisiones distintas de la familia Pano. La ubicación precisa de la lengua iskonawa no ha sido establecida ${ }^{31}$.

\subsection{Posible Escenario Histórico}

En esta sección se delinea el posible desarrollo diacrónico de los morfemas de $\mathrm{CP}$ en las lenguas Pano considerando seis etapas.

\section{Etapa 1: Marcadores de caso en la lengua antecesora}

La lengua antecesora habría tenido, en alguna parte de su gramática, un sistema de caso tripartito con marcadores similares a los que exhibe el kashibokakataibo actual:

${ }^{*}-n$ for $\mathrm{A},{ }^{*}-\mathrm{s}$ for $\mathrm{S}$, and ${ }^{*}-0$ for $\mathrm{O}$.

Es probable que este sistema tripartito no haya operado de una manera tan extensa como ocurre en kashibo-kakataibo, sino que más bien haya estado limitado a contextos pragmáticamente marcados. Además, los siguientes marcadores periféricos o (no) secuenciales habrían existido en la Proto-lengua:

${ }^{*}$-so SEC, ${ }^{*}$-?a SEC/COMPL, ${ }^{*}-k i$ LOC APROX, ${ }^{*}$-no LOC/DIR

31 Valenzuela (2003) nota que mientras Loos (1999) clasifica a esta lengua conjuntamente con el kapanawa y shipibo-konibo, d'Ans (1973) la coloca en su grupo de las Cabeceras donde también se encuentran el yaminawa y sharanawa (y también el amawaka). 


\section{Etapa 2: Añadidura de marcadores periféricos a cláusulas nominalizadas}

Los marcadores de caso periféricos o (no) secuenciales son añadidos a cláusulas nominalizadas para codificar relaciones temporales o lógicas entre dos eventos o circunstancias que probablemente compartían un argumento tópico. En este contexto se habría producido la elisión del sujeto compartido (equi-subject deletion). La semántica de los marcadores periféricos es compatible con las relaciones temporales o lógicas entre los eventos.

Marcadores Originales y Funciones

${ }^{*}$-so $\sim{ }^{*}$-su SEC

${ }^{*}-? a \quad$ SEC/COMPL

* $-k i \quad$ LOC APROX

${ }^{*}-n o \sim{ }^{*}-n u \quad$ LOC/DIR
Nuevas Funciones

Evento previo: 'después de', 'y', 'porque' Evento previo: 'después de', 'y', 'porque' Evento simultáneo: 'mientras', 'durante' Evento posterior: 'antes de', 'para'

Especialmente los marcadores locativos han retenido su función original en las lenguas actuales.

Etapa 3: Reinterpretación de los marcadores de caso periféricos o (no) secuenciales

Los marcadores agregados a las cláusulas nominalizadas son reinterpretados como morfemas de correferencialidad de sujeto. Posiblemente, -so $\sim-s u,-? a$, y - ki mostraban cierta distribución complementaria. Por ejemplo, $-s o \sim-s u$ habría alternado con -?a; nótese que ninguna lengua Pano combina $-s o \sim-s u+$ $-s$, ni tampoco $-2 a+-n$; similarmente, ninguna lengua muestra la secuencia $-k i+s$.

Las distribuciones complementarias mencionadas arriba habrían estado asociadas al hecho de que, además de codificar relaciones lógico-temporales e identidad de sujetos, los marcadores en cuestión tienden a restringir la función sintáctica del argumento correferencial en la cláusula matriz. Recuérdese que al hablar de "sujeto" se debe distinguir A de S.

Marcador de SI Restricción de correferencialidad

-so -su A (aunque amawaka - xo y matsés $-\int 0>0$ )

$-2 a \quad \mathrm{~S}$ (aunque algunas lenguas $-a$ y matsés $-a k \mathrm{O}>\mathrm{S} / \mathrm{A}$ )

$-k i \quad$ A (ausente en kapanawa)

-no -nu S/A (shipibo-konibo, yaminawa, kashibo-kakataibo, matsés)

Además, el locativo -no -nu se emplea para marcar sujeto-diferente en algunas lenguas como el yaminawa y el shipibo-konibo.

Etapa 4: Añadidura de los marcadores argumentales

${ }^{*}-n,{ }^{*}-S, \mathrm{y}{ }^{*}-0$ se añaden a las cláusulas nominalizadas (que ya llevan marcadores periféricos) en concordancia con la función sintáctica del 
argumento correferencial en la cláusula matriz. Probablemente estos marcadores contribuyeron a la codificación del seguimiento de la referencia, permitiendo, por ejemplo, el contraste entre - $x o$ vs. - $x o-n$ en Amawaka, $-a$ vs. - as en shipibo-konibo y -nu vs. -nun en matsés.

\section{Etapa 5: Fusión y expansión de los marcadores de sujeto-idéntico}

Las secuencias de marcadores pertenecientes a las dos capas descritas arriba se fusionan, formándose morfemas portmanteau que codifican simultáneamente correferencialidad de sujeto, orden relativo de eventos y la función sintáctica del argumento controlador en la cláusula matriz.

Luego, algunas cláusulas adjuntas, posiblemente con copula cero o con verbos (auxiliares) semánticamente genéricos son reinterpretados como frases en vez de cláusulas; consecuentemente, las construcciones de dos cláusulas de las que participaban son reanalizadas como construcciones de una sola cláusula. Esto es particularmente evidente cuando se examinan los adjuntos de manera, razón y cantidad (véase los adjuntos en las oraciones (64) del shipibo-konibo y (65) del kashibo-kakataibo). Posteriormente, los marcadores de sujetos idénticos se expanden a ciertas frases o palabras adjuntas (e.g. afectivos en shipibo-konibo y yaminawa).

\section{Etapa 6: Pérdida de la marcación de sujeto intransitivo}

En la mayoría de las lenguas la marcación de sujeto intransitivo se pierde en micro-alineamientos que exhibían una configuración tripartita: - $\mathrm{s}>-0$ (es posible que en kashibo-kakataibo el alineamiento tripartito se habría más bien ampliado). Consecuentemente, $\mathrm{S}$ es marcado de la misma forma que $\mathrm{O}$ y diferentemente de A, resultando en un alineamiento ergativo-absolutivo. Sin embargo, la distribución tripartita es retenida en los marcadores de $\mathrm{CP}$ en los adjuntos, lo cual puede ser visto como un tipo muy peculiar de ergatividad escindida (Valenzuela 2003:929).

Algunas lenguas pierden el marcador secuencial $-s o \sim-s u$. En shipibokonibo y posiblemente otras lenguas, $-n o \sim-n u>-n$; la forma no reducida se retiene en contextos fijos como por ejemplo ningo 'aquí', ono por allá', actualmente no analizables en shipibo-konibo.

\section{Posible desarrollo alternativo}

Cuando están orientados a $\mathrm{S}$, algunos adjuntos llevan el marcador $-s$ de forma directa, es decir sin requerir del formante -?a. Una instancia de esto lo constituye $\beta a k i-s$ [niño-S] 'de niño', en el ejemplo del shipibo-konibo. Estos datos sugieren la posibilidad de un desarrollo distinto para ciertos adjuntos, simplemente basado en la concordancia de caso. Es interesante que en estos 
casos estemos hablando de tipos de adjuntos con un alto grado de orientación hacia el participante (Valenzuela 2005a también registra este patrón con los pronombres enfáticos). Sin embargo, cuando están orientados hacia A los marcadores de etapa de vida llevan la forma -son en lugar de - $n$ (véase el ejemplo ). Probablemente, este desarrollo tuvo lugar cuando el marcador -son ya no era analizable ${ }^{32}$ (véase también la forma shipibo-konibo -no-son en vez de - non).

\section{Comentarios finales}

Este artículo ha presentado un análisis sincrónico y diacrónico del sistema de concordancia del participante (CP) en las lenguas Pano tomando como base Valenzuela (2003:capítulos 19 y 20; 2005a); asimismo, se ha echado mano de los aportes descriptivos que se han hecho disponibles durante los últimos años, específicamente en lo que respecta a los sistemas de marcación de caso y de CP. Se ha demostrado que la CP opera en dos niveles sintácticos: en frases adjuntas al interior de la cláusula y en cláusulas dependientes al concatenar cláusulas. En este último contexto sintáctico, los morfemas de $\mathrm{CP}$ forman parte del sistema de cambio de referencia (switch-referfence), funcionando especialmente como indicadores de sujetos idénticos y otros tipos de correferencia (por ejemplo, $\mathrm{O}>\mathrm{S} / \mathrm{A}$ y $\mathrm{S} / \mathrm{A}>\mathrm{O}$ ). En cuanto a la distribución de los morfemas de $\mathrm{CP}$, se ha demostrado que esta depende de la función sintáctica del participante acerca del cual el adjunto predica, y no de la transitividad del verbo (matriz) en sí. Es decir,, la distribución de los morfemas de $\mathrm{CP}$ se correlaciona con la transitividad, mas no está determinada por esta. Esto es evidente al examinar los casos de concordancia intra-oracional donde un mismo predicado transitivo puede coocurrir con un adjunto orientado hacia el participante A (marcado por -son -sun) o con un adjunto orientado hacia el participante $\mathrm{O}$ (marcado por -0). Por lo tanto, no es acertado referirse a este sistema con el rótulo de "concordancia transitiva".

Como se ha mostrado en este artículo, tanto por sus peculiaridades sincrónicas (secciones 2 y 3), así como por la trayectoria que habría seguido su desarrollo diacrónico (sección 4), la CP Pano es un rasgo gramatical único, de gran interés para la teoría y tipología lingüísticas, que requiere ser descrito detallada y exhaustivamente en cada uno de los idiomas. Pero además, el estudio de la $\mathrm{CP}$ es importante por las implicancias que tiene para avanzar nuestro conocimiento de la gramática Proto-Pano. Dado que la $\mathrm{CP}$ está presente en todas las lenguas consideradas en este trabajo, representantes de las diferentes

32 No obstante, debemos señalar que el agregar directamente a $\beta a k i$ el marcador de caso correspondiente a A resultaría en una forma idéntica a la ergativa, lo cual podría dificultar la decodificación de la expresión. Recuérdese que a diferencia de $-n,-\$$ ya no existe como marcador de caso en shipibo-konibo. 
subdivisiones de la familia Pano, y que los marcadores involucrados son (casi) idénticos en forma y función, es posible postular que estamos frente a un rasgo que formaba parte de la proto-lengua. También se ha sostenido que el sistema de CP Pano está diacrónicamente relacionado a un proceso de concordancia de caso en cláusulas nominalizadas y que la lengua antecesora habría exhibido un sistema de marcación de caso tripartito en algún ámbito de su gramática. El material fonético de los marcadores de caso habría sido muy similar al que encontramos actualmente en el kashibo-kakataibo. Una interrogante pendiente concierne el ámbito preciso de operación del patrón tripartito en Proto-Pano. Es probable que este haya sido menos extendido que el atestiguado actualmente en kashibo-kakataibo, habiendo estado restringido a contextos pragmáticamente marcados como ocurre en amawaka. En efecto, entre sus "reglas transformacionales peculiares" de las lenguas Pano Loos (1973:270) menciona la "Permutación del sujeto del verbo a posición posverbal en el aspecto incompleto" (reminiscente del amawaka y chákobo), así como la "Consignación de la marca de intransitividad al sujeto del verbo". Posteriormente, la marca de sujeto intransitivo (es decir ${ }^{*}-s$ ) habría sido abandonada (Loos 1973:273). Lamentablemente, esta interesante propuesta de Loos adolece de la falta de datos lingüísticos suficientes que sirvan de sustento a su hipótesis. Por último, es importante notar que Valenzuela (2003 y este artículo) arriba a la misma conclusión con respecto a la existencia de un sistema de marcación de caso tripartito en Proto-Pano en base al análisis de la CP.

\section{Referencias}

Amarante Ribeiro, Lincoln. 2005. Uma proposta de classificaçaõ interna das línguas da familia pano. Investigações, pp. 157-182.

D’Ans, Marcel. 1973. Reclasificación de las lenguas pano y datos glotocronológicos para la etnohistoria de la Amazonía peruana. Revista del Museo Nacional, tomo XXXIX:349-369.

Córdoba, Lorena, Pilar Valenzuela \& Diego Villar. Pano Meridional. En M. Crevels \& P. Muysken (eds.), Las lenguas de Bolivia, Tomo II: Amazonía. La Paz, Bolivia: Plural Editores, pp. 27-69.

Dixon, R.M.W. 1994. Ergativity. Cambridge: Cambridge University Press.

Erikson, Philipe. 1999. El sello de los antepasados. Marcado del cuerpo y demarcación étnica entre los matis de la Amazonía. Quito: Abya-Yala e Instituto Francés de Estudios Andinos.

Erikson, Philippe, Bruno Illius, Kenneth Kensinger y Maria Suelli de Aguiar. 2004. Kirinkobaon kirika (“Gringo's books"); an annotated Panoan bibliography. Amerindia, supplément 1 au n.19. Paris: CNRS. 
Fabre, A. 2005. Pano-Takana. En Diccionario etnolingüístico y guía bibliográfica de los pueblos indígenas sudamericanos: http://butler.cc.tut.fi/ fabre/BookInternetVersio/ Dic=PanoTakana.pdf

Faust, Norma y Eugene Loos. 2002. Gramática del idioma yaminahua. Lima: Instituto Lingüístico de Verano.

de Figueroa, Francisco. [1661] 1904. Relación de las misiones de la Compañía de fesús en el país de los Maynas.Madrid: Libr. General de Victoriano Suárez (Colección de Libros y Documentos referentes a la Historia de América, Tomo I).

Fleck, D. W. 2003. A grammar of Matses. Ph.D. dissertation, Rice University, Houston.

Fleck, D. W. 2010. Ergativity in the Mayoruna branch of the Panoan family. En S. Gildea y F. Queixalós (eds.) Ergativity in Amazonia, Amsterdam: John Benjamins, pp. 29-63.

Fleck, D. W. de pronta aparición. Panoan languages and linguistics. Anthropological Papers of the American Museum of Natural History.

Fleck Zuazo, D., F. Sh. Uaquí Bëso y D. M. Jiménez Huanán. 2012. Diccionario matséscastellano. Iquitos: Tierra Nueva.

Grasserie, Raoul de la. 1890. De la famille linguistique Pano. Compte-rendu de la Septième Session du Congrès International des Américanistes. Berlin 1888, pp. 438462. Berlín: W. H. Kuhl.

Himmelmann, N. y E. Schultze-Berndt. 2005. Issues in the syntax and semantics of participant-oriented adjuncts: an introduction. En N. Himmelmann y E. SchultzeBerndt (eds.), Secondary predication and adverbial modification: crosslinguistic explorations in the syntax and semantics of depictives. Oxford: Oxford University Press, pp. 1-67.

Instituto Nacional de Estadística e Informática. 2007. Resumen Ejecutivo Resultados Definitivos de los Censos en Comunidades Indígenas de la Amazonía Peruana. Consulta: 28 de julio de 2011. <http://censos.inei.gob.pe/Censos2007/PagCensos_ ResultadosComunidadesIndigenas1.asp $>$.

Kneeland, Harriet. 1979. Lecciones para el aprendizaje del idioma mayoruna. Yarinacocha: Instituto Lingüístico de Verano y Ministerio de Educación.

Levy, Carlos Daniel. 1991. The notion of a nation: the Shipibo-Conibo. Ponencia presentada al 47 Congreso Internacional de Americanistas, New Orleans.

Lewis, M. P. (ed.), 2009. Ethnologue: Languages of the World, Sixteenth edition. Dallas, Tex.: SIL International. Online version: http://www.ethnologue.com/.

Loos, Eugene E. 1973. Algunas implicancias de la reconstrucción de un fragmento de la gramática del proto-pano. En Eugene E. Loos (ed.) Estudios Panos II, Serie Lingüística Peruana 11, Yarinacocha, Perú: Ministerio de Educación e Instituto Lingüístico de Verano, pp. 263-282.

. 1978. La señal de transitividad del sustantivo en los idiomas panos. En Ministerio de Educación e ILV (eds.) Estudios Panos I, Serie Lingüística Peruana 10 (segunda edición), pp. 133-184. 
1999. Pano. En R.M.W. Dixon y A.Y. Aikenvald (eds.), The Amazonian Languages, Cambridge: Cambridge University Press, pp. 227-250.

.2005. Un breve estudio de la gramática del proto-pano. Revista Latinoamericana de Estudios Etnolingüísticos, pp. 11: 37-50.

Montag, Richard. 2005. Participant referencing in Cashinahua. SIL Electronic Working Papers 2005-013. S.l.: SIL International. 11 páginas. http://www.sil.org/silewp/ abstract.asp?ref=2005-013

Prost, Gilbert R. 1965. Chácobo. En Gramáticas estructurales de lenguas bolivianas 2, Esther Matteson (ed.), pp. 1-130. Riberalta: Instituto Lingüístico de Verano.

Schultze-Berndt, Eva y Nikolaus. P. Himmelmann. 2004. Depictive secondary predicates in cross-linguistic perspective. Linguistic Typology 8,1:59-131.

Shell, Olive. 1975[1965]. Las lenguas pano y su reconstrucción, Estudios Pano III. Pucallpa: Ministerio de Educación-Instituto Lingüístico de Verano.

Sparing-Chávez. M. 1998. Interclausal reference in Amahuaca. En Derbyshire y Pullum (eds.), Handbook of Amazonian Languages, vol. 4, Berlin/New York: Mouton de Gruyter, pp. 443-485.

Sorensen, Arthur P. 1969. Multilingualism in the Northwest Amazon. American Anthropologist 69, 6:670-684.

Tessmann, Günter. 1999[1930]. Los indios del Perú nororiental. Investigaciones fundamentales para un estudio sistemático de la cultura. Quito: Abya Yala.

Valenzuela, Pilar M. 1999. Adverbials, Transitivity and Switch-Reference in ShipiboKonibo (Panoan). En S.J. Billings, J.P. Boyle y A.M. Griffith (eds.), Chicago Linguistic Society 35: The Panels, Theory and Linguistic Diversity, Chicago: Chicago Linguistic Society, pp. 355-371.

. 2000. "Ergatividad escindida en wariapano, yaminawa y shipibo-konibo". En Hein van der Voort and Simon van de Kerke (eds.), Indigenous languages of Lowland South America. Indigenous Languages of Latin America (ILLA) 1. Leiden, The Netherlands: University of Leiden, pp. 111-128

. 2002. Causativization and Transitivity in Shipibo-Konibo. En Masayoshi Shibatani (ed.), The Grammar of Causation and Interpersonal Manipulation, Typological Studies in Language. Amsterdam: John Benjamins. pp. 417-483.

. 2003. Transitivity in Shipibo-Konibo grammar. Ph.D. dissertation, University of Oregon.

. 2005a. Participant-oriented adjuncts in Panoan. En Nikolaus P. Himmelmann y Eva Schultze-Berndt (eds.), Secondary predication and adverbial modification: The typology of depictives, Oxford: Oxford University Press, pp. 259-298.

2005b. Adjuntos Orientados hacia un Participante en Chacobo (Pano). Acercamiento Comparativo y Tipológico. UniverSOS. Valencia: Guada Impresores, pp. 185-200. 
. 2011. Multi-verb predicates and transitivity harmony in Shipibo-Konibo. En P. C. Muysken y A. Y. Aikhenvald (eds.), Multi-verb Constructions: A view from the Americas. Studies in the Indigenous Languages of the Americas, Amsterdam: Brill, pp. 185-212.

Valenzuela, Pilar M. y Oliver A. Iggesen. 2007. El desarrollo de un marcador suprasegmental en chácobo (pano). En A. Romero Figueroa, A. Fernández Garay y A. Corbera Mori (eds.), Lenguas indígenas de América del Sur: Estudios descriptivotipológicos y sus contribuciones para la lingüística teórica, 187-199. Caracas: Universidad Católica "Andrés Bello".

Zariquiey Biondi, Roberto. 2011. A Grammar of Kashibo-Kakataibo. Ph.D. dissertation, La Trobe University, Australia.

Zingg, Philipp (1998). Diccionario Chacobo-Castellano y Castellano-Chacobo. La Paz: Ministerio de Desarrollo Sostenible y Planificación-Viceministerio de Asuntos Indígenas y Pueblos Originarios.

Data recebimento: $19 / 02 / 2013$

Data aceite: 27/05/2013 